\title{
Application of an extended random-phase approximation to giant resonances in light-, medium-, and heavy-mass nuclei
}

\author{
V. Tselyaev and N. Lyutorovich \\ St. Petersburg State University, 7/9 Universitetskaya nab., St. Petersburg, 198504, Russia \\ J. Speth ${ }^{*}$ and S. Krewald \\ Institut für Kernphysik, Forschungszentrum Jülich, D-52425 Jülich, Germany \\ P.-G. Reinhard \\ Institut für Theoretische Physik II, Universität Erlangen-Nürnberg, D-91058 Erlangen, Germany
}

(Received 3 March 2016; published 6 September 2016)

\begin{abstract}
We present results of the time blocking approximation (TBA) for giant resonances in light-, medium-, and heavy-mass nuclei. The TBA is an extension of the widely used random-phase approximation (RPA) adding complex configurations by coupling to phonon excitations. A new method for handling the single-particle continuum is developed and applied in the present calculations. We investigate in detail the dependence of the numerical results on the size of the single-particle space and the number of phonons as well as on nuclear matter properties. Our approach is self-consistent, based on an energy-density functional of Skyrme type where we used seven different parameter sets. The numerical results are compared with experimental data.
\end{abstract}

DOI: 10.1103/PhysRevC.94.034306

\section{INTRODUCTION}

Self-consistent mean-field models have been developed over the decades into a powerful tool for the description of nuclear structure and dynamics all over the periodic table [1-4]. Time-dependent mean-field theory allows us to simulate a great variety of excitations and dynamical processes [5]. Giant resonances are described well in the small amplitude limit where the space of one-particle-one-hole (1p-1h) excitations is explored, which is, in fact, identical to the widely used random phase approximation (RPA). Here one is able to calculate mean energies and total transition strengths. In order to describe also the fine structure of bound states and the total width of giant resonances one has to include correlations beyond $1 \mathrm{p}-1 \mathrm{~h}$. Such calculations have been performed in self-consistent as well as in non-self-consistent approaches. Extended theories may include, e.g., two-particle-two-hole configurations [6] or one may consider the fragmentation of the single-particle states due to the coupling to phonons [7-10]. Within the latter approach isoscalar electric monopole resonances and quadrupole resonances were well reproduced in medium- and heavy-mass nuclei [9-13]. In light nuclei like ${ }^{16} \mathrm{O}$ the present theory is unable to reproduce the experimental isoscalar cross sections quantitatively, as important decay channels are still missing. This will be discussed in Sec. III.

One might assume that mean-field theories which describe bulk properties of nuclei, such as the Thomas-Reiche-Kuhn (TRK) sum rule and the nuclear symmetry energy [14], as well as shell effects, should also reproduce rather well the centroid energies of the giant dipole resonance (GDR). This is not the case, however, as has been worked out in systematic surveys based on RPA spectra [15-17]. It was impossible

"J.Speth@fz-juelich.de to describe ground-state properties and the centroid energy of the GDR both in light and heavy nuclei with the same effective interaction. The problem is more serious than it might appear at a first glance because the physics of the GDR is closely connected with the neutron skin thickness and the low-lying dipole strength: the so-called pygmy resonances [18-20]. These states are presently investigated experimentally because of their impact on the isotope abundance produced in supernova explosions [21].

Recently we showed that the explicit inclusion of quasi particle-phonon coupling may help to solve the problem of mean-field theories in reproducing the centroid energies of the GDR [22]. Within the time blocking approximation (TBA) $[8,9]$, i.e., including $1 \mathrm{p}-1 \mathrm{~h} \otimes$ phonon configurations explicitly, we obtained a reasonably good quantitative agreement with the experimental data for the GDR in light $\left({ }^{16} \mathrm{O}\right)$, medium $\left({ }^{48} \mathrm{Ca}\right)$, and heavy $\left({ }^{208} \mathrm{~Pb}\right)$ nuclei. As we went beyond the mean-field approach we had to adjust new Skyrme forces, where we concentrated on the GDR in ${ }^{16} \mathrm{O}$ within the conventional $1 \mathrm{p}-1 \mathrm{~h}$ RPA. If we confine ourselves in the TBA to the contribution of the most collective phonons, the TBA hardly changed the $1 \mathrm{p}-1 \mathrm{~h}$ RPA result in ${ }^{16} \mathrm{O}$ but moved the GDR in ${ }^{48} \mathrm{Ca}$ and ${ }^{208} \mathrm{~Pb}$ closer to the experimental values. The isoscalar giant monopole resonance (GMR) and giant quadrupole resonance (GQR) were shown in a short note [10] using an improved version of TBA that derived all matrix elements consistently from the given (Skyrme) energy-density functional and calculated them without any approximations and included the single-particle continuum, thus avoiding the artificial discretization implied in earlier TBA calculations. The present publication discusses in detail the formalism of the short note [10]. Moreover, we present a new treatment of the single-particle continuum which allows us (i) to include exactly the velocity dependent terms and the spin-orbit interaction and (ii) to eliminate the unphysical 
effects generated in the $1 \mathrm{p}-1 \mathrm{~h} \otimes$ phonon configurations when employing a discretized single-particle basis.

We scrutinize the phonon-coupling model by studying the dependence of the results on the numerical parameters of the model (more formal details were presented recently in [23]). The theoretical spectral distributions for the GMR, GQR, and GDR of ${ }^{16} \mathrm{O},{ }^{40} \mathrm{Ca},{ }^{48} \mathrm{Ca}$, and ${ }^{208} \mathrm{~Pb}$ are compared with the experimental ones. We use seven different Skyrme parameter sets in order to find out how these giant resonances depend on some specific gross properties of nuclear matter.

The paper is organized as follows. In Sec. II A we present the basic formulas of the self-consistent RPA and TBA. In Sec. II B we present seven different Skyrme parameter sets which reproduce the usual ground-state properties and give reasonably good results for isovector as well as isoscalar electric giant resonances. The Skyrme parameter sets were characterized in terms of nuclear matter properties (NMPs) from which we consider in particular four key quantities: incompressibility $K$, effective mass $m^{*} / m$, symmetry energy, and enhancement factor for the TRK sum rule $\kappa_{\mathrm{TRK}}$ (equivalent to isovector effective mass). We investigated in detail the influence of these four NMPs on the GDR and the giant isoscalar monopole and quadrupole resonances. Problems connected with the tuning of the parameters are discussed in Sec. II C. Details of the calculation scheme are given in Sec. III. In Sec. III A we discuss the single-particle basis and in Sec. III B the effect of the exact continuum treatment on our results. In Sec. III C we investigate in detail the dependence of the TBA results on the number of phonons included. Section IV presents our results. In Sec. IV A we compare our final results with experimental data, and we analyze the results in Sec. IV B. In the last section we summarize our investigations.

\section{THE METHOD}

\section{A. The basic equations}

\section{Conventional RPA}

The original derivation of the RPA equations in nuclear physics is based on the time-dependent Hartree-Fock methods where one considered small amplitude dynamics about a Hartree-Fock ground state [24]. From this derivation, one may obtain the impression that the RPA is a very limited approach. This is actually not the case if one considers the derivation within the Green function method. All details and the explicit expressions can be found in Ref. [25]. The transition matrix element of a one-particle operator between the exact ground state of an $A$-particle system and an excited state $m$ is given as

$$
\langle A m|Q| A 0\rangle=\sum_{v_{1} v_{2}} Q_{v_{1} v_{2}}^{\mathrm{eff}} \chi_{v_{1} \nu_{2}}^{m} .
$$

Here $\mathrm{Q}^{\text {eff }}$ are effective operators and $\chi^{m}$ are the quasiparticlequasihole matrix elements which are given by the equation

$$
\left(\epsilon_{v_{1}}-\epsilon_{\nu_{2}}-\Omega\right) \chi_{\nu_{1} \nu_{2}}^{m}=\left(n_{v_{1}}-n_{\nu_{2}}\right) \sum_{\nu_{3} v_{4}} F_{\nu_{1} \nu_{4} \nu_{2} \nu_{3}}^{\mathrm{ph}} \chi_{\nu_{3} \nu_{4}}^{m}
$$

where $\mathrm{F}^{\mathrm{ph}}$ is the renormalized particle-hole interaction. All relations have been been derived without any approximations.
Therefore conservation laws can be applied. For example, the effective electric operators reduce to the bare ones due to Ward identities in the long-wavelength limit. The derivation of the RPA equation starts with the equation of motion (Dyson equation) for the one-particle Green function. The basic input is the mass operator $\Sigma$ which includes all information on the many-body system. The most general form is given as

$$
\Sigma=\Sigma(\mathbf{r}, \mathbf{p}, \epsilon)
$$

It depends on the coordinate $\mathbf{r}$, the momentum $\mathbf{p}$ (non-locality), and the energy $\epsilon$.

Note that the RPA equations derived here are formally identical with the corresponding equations derived in the linear response limit of time-dependent density-functional theory (TDDFT) in the next section. The crucial difference is the mass operator in Eq. (3) which is energy dependent in a general many-body theory whereas it turns out to be independent of energy in TDDFT. As the various quantities in the general case and in linear response are different, we also use different symbols.

In the general case, the expression for the effective mass has the form

$$
\frac{m}{m^{*}}=\frac{\left(1+2 m \frac{\delta \Sigma}{\delta p^{2}}\right)_{F}}{\left(1-\frac{\delta \Sigma}{\delta \epsilon}\right)_{F}}
$$

The nominator is called $k$ mass and the denominator $E$ mass [26]. They are related to the nonlocality and energy dependence of the mass operator, respectively. If the mass operator does not depend on the energy, the denominator is equal to 1 . In the case of a totally energy-independent mass operator, the formulas become much simpler as the single-particle strength is equal to one [27]. The effective operators are in all cases equal to the bare operators and also the particle-hole interaction is not renormalized.

In our extended model (the TBA), we introduce complex configurations by coupling phonons to the single-particle states. This introduces an energy dependence into the mass operator in first order [28]. For this reason the single-particle strength is less then 1 and we obtain a contribution to the $E$ mass. This is the well known shift due to phonon coupling. All this is correctly taken care of in the TBA. But we will not address single-particle effects explicitly later on.

\section{Self-consistent RPA}

Our approach is based on the version of the response function formalism developed within the Green function method (see Ref. [25]). In the general case the distribution of the strength of transitions in the nucleus caused by some external field represented by the single-particle operator $Q$ is determined by the strength function $S(E)$ which is defined in terms of the response function $R(\omega)$ by the formulas

$$
\begin{aligned}
& S(E)=-\frac{1}{\pi} \operatorname{Im} \Pi(E+i \Delta), \\
& \Pi(\omega)=-\langle Q|R(\omega)| Q\rangle,
\end{aligned}
$$

where $E$ is an excitation energy, $\Delta$ is a smearing parameter, and $\Pi(\omega)$ is the (dynamic) polarizability. 
The first model used in our calculations is the self-consistent RPA based on TDDFT with the energy density functional $E[\rho]$. The TDDFT equations imply that $[\rho, h]=0$ where $\rho$ is the single-particle density matrix satisfying the condition $\rho^{2}=\rho$, and $h$ is the single-particle Hamiltonian,

$$
h_{12}=\frac{\delta E[\rho]}{\delta \rho_{21}} .
$$

The numerical indices here and in the following denote the set of the quantum numbers of some single-particle basis. It is convenient to introduce the basis that diagonalizes simultaneously the operators $h$ and $\rho$ :

$$
h_{12}=\varepsilon_{1} \delta_{12}, \quad \rho_{12}=n_{1} \delta_{12},
$$

where $n_{1}=0,1$ is the occupation number. In what follows the indices $p$ and $h$ will be used to label the single-particle states of the particles $\left(n_{p}=0\right)$ and holes $\left(n_{h}=1\right)$ in this basis.

In RPA, the response function is a solution of the following Bethe-Salpeter equation (BSE):

$$
R^{\mathrm{RPA}}(\omega)=R^{(0)}(\omega)-R^{(0)}(\omega) V R^{\mathrm{RPA}}(\omega),
$$

where $R^{(0)}(\omega)$ is the uncorrelated $1 \mathrm{p}$ - $1 \mathrm{~h}$ propagator and $V$ is the residual interaction. The $1 \mathrm{p}-1 \mathrm{~h}$ propagator $R^{(0)}(\omega)$ is defined as

$$
R^{(0)}(\omega)=-\left(\omega-\Omega^{(0)}\right)^{-1} M^{\mathrm{RPA}},
$$

where the matrices $\Omega^{(0)}$ and $M^{\text {RPA }}$ are defined in the $1 \mathrm{p}-1 \mathrm{~h}$ configuration space. $M^{\mathrm{RPA}}$ is the metric matrix

$$
M_{12,34}^{\mathrm{RPA}}=\delta_{13} \rho_{42}-\rho_{13} \delta_{42} .
$$

The matrix $\Omega^{(0)}$ has the form

$$
\Omega_{12,34}^{(0)}=h_{13} \delta_{42}-\delta_{13} h_{42} .
$$

In the self-consistent RPA based on the energy density functional $E[\rho]$ one has

$$
V_{12,34}=\frac{\delta^{2} E[\rho]}{\delta \rho_{21} \delta \rho_{34}},
$$

so the quantities $h$ and $V$ appear to be linked by Eqs. (7) and (13).

The propagator $R^{\mathrm{RPA}}(\omega)$, being a matrix in $1 \mathrm{p}-1 \mathrm{~h}$ space, is a rather bulky object. For practical calculations, it is more convenient to express it in terms of RPA amplitudes $z_{12}^{n}$ by virtue of the spectral representation

$$
R_{1234}^{\mathrm{RPA}}(\omega)=-\sum_{n} z_{12}^{n} \frac{\operatorname{sgn}\left(\omega_{n}\right)}{\omega-\omega_{n}}\left(z_{34}^{n}\right)^{*},
$$

where $n$ labels the RPA eigenmodes and $\omega_{n}$ is the eigenfrequency. Inserting that into Eq. (9) and filtering the pole at $\omega=\omega_{n}$ yields the familiar RPA equations

$$
\sum_{34}\left(\Omega_{12,34}^{(0)}+\sum_{56} M_{12,56}^{\mathrm{RPA}} V_{56,34}\right) z_{34}^{n}=\omega_{n} z_{12}^{n},
$$

where the transition amplitudes $z^{n}$ are normalized by the condition

$$
\sum_{12,34}\left(z_{12}^{n}\right)^{*} M_{1234}^{\mathrm{RPA}} z_{34}^{n^{\prime}}=\operatorname{sgn}\left(\omega_{n}\right) \delta_{n, n^{\prime}} .
$$

These equations determine the set of eigenstates $n$ with amplitudes $z_{12}^{n}$ and frequencies $\omega_{n}$.

\section{Phonon coupling model}

The second model is the quasiparticle-phonon coupling model within the time-blocking approximation (TBA) $[8,9,12,29]$ (without ground state correlations beyond the RPA included in $[8,9,12,29]$ and without pairing correlations included in [9]). This model, which in the following will be referred to as TBA, is an extension of RPA including $1 \mathrm{p}-1 \mathrm{~h} \otimes$ phonon configurations in addition to the $1 \mathrm{p}-1 \mathrm{~h}$ configurations incorporated in the conventional RPA. The BSE for the response function in the TBA is

$$
\begin{aligned}
R^{\mathrm{TBA}}(\omega) & =R^{(0)}(\omega)-R^{(0)}(\omega)[V+\tilde{W}(\omega)] R^{\mathrm{TBA}}(\omega), \\
\tilde{W}(\omega) & =W(\omega)-W(0),
\end{aligned}
$$

where the induced interaction $\tilde{W}(\omega)$ serves to include contributions of $1 \mathrm{p}-1 \mathrm{~h} \otimes$ phonon configurations.

The matrix $W(\omega)$ in Eq. (18) is defined in the $1 \mathrm{p}-1 \mathrm{~h}$ subspace and can be represented in the form

$$
W_{12,34}(\omega)=\sum_{c, \sigma} \frac{\sigma F_{12}^{c(\sigma)} F_{34}^{c(\sigma) *}}{\omega-\sigma \Omega_{c}},
$$

where $\sigma= \pm 1, c=\left\{p^{\prime}, h^{\prime}, n\right\}$ is an index of the subspace of $1 \mathrm{p}-1 \mathrm{~h} \otimes$ phonon configurations, $n$ is the phonon's index,

$$
\begin{aligned}
\Omega_{c} & =\varepsilon_{p^{\prime}}-\varepsilon_{h^{\prime}}+\omega_{n}, \quad \omega_{n}>0, \\
F_{12}^{c(-)} & =F_{21}^{c(+) *}, \quad F_{\mathrm{ph}}^{c(-)}=F_{h p}^{c(+)}=0, \\
F_{\mathrm{ph}}^{c(+)} & =\delta_{p p^{\prime}} g_{h^{\prime} h}^{n}-\delta_{h^{\prime} h} g_{p p^{\prime}}^{n},
\end{aligned}
$$

and $g_{12}^{n}$ is an amplitude of the quasiparticle-phonon interaction. These $g$ amplitudes (along with the phonon's energies $\omega_{n}$ ) are determined by the positive frequency solutions of the RPA equations and the emerging $z$ amplitudes as

$$
g_{12}^{n}=\sum_{34} V_{12,34} z_{34}^{n}
$$

where $V_{12,34}$ is the same residual interaction (13) as used in RPA. In our DFT-based approach the energy density functional $E[\rho]$ in Eqs. (7) and (13) is the functional of the Skyrme type with free parameters which are adjusted to experimental data. In this case $E[\rho]$ already effectively contains a part (actually the stationary part) of the contributions of those $1 \mathrm{p}-1 \mathrm{~h} \otimes$ phonon configurations which are explicitly included in the TBA. Therefore, in the theory going beyond the RPA, the problem of double counting and of ground-state stability arises [30]. To avoid this problem in the TBA, we use the subtraction method. It consists of the replacement of the amplitude $W(\omega)$ by the quantity $\bar{W}(\omega)=W(\omega)-W(0)$ as given in Eq. (17). In Ref. [31] it was shown that, in addition to the elimination of double counting, the subtraction method ensures stability of solutions of the TBA eigenvalue equations. 


\section{B. Basics on the Skyrme functional and related parameters}

From the variety of self-consistent nuclear mean-field models [2], we consider here a nonrelativistic branch, the widely used and very successful Skyrme-Hartree-Fock (SHF) functional. A detailed description of the functional is found in the reviews $[2,16,32]$. We summarize the essential features: The functional depends on a few local densities and currents (density, gradient of density, kinetic-energy density, spin-orbit density, current, spin density, and kinetic spin density). It consists of quadratic combinations of these local quantities, corresponding to pairwise contact interactions. The term with the local densities is augmented by a nonquadratic density dependence to provide appropriate saturation. One adds a simple pairing functional to account for nuclear superfluidity. The typically 13-14 model parameters are determined by a fit to a large body of experimental data on bulk properties of the nuclear ground state. For recent examples see $[3,4,15]$.

The properties of the forces can be characterized, to a large extent, by nuclear matter properties (NMPs), i.e., equilibrium properties of homogeneous, symmetric nuclear matter, for which we have some intuition from the liquid-drop model [33]. Of particular interest for resonance excitations are the NMPs which are related to response to perturbations: incompressibility $K$ (isoscalar static), effective mass $\mathrm{m}^{*} / \mathrm{m}$ (isoscalar dynamic), symmetry energy $a_{\text {sym }}$ (isovector static), and TRK sum rule enhancement $\kappa_{\mathrm{TRK}}$ (isovector dynamic). We aim at exploring the effect of phonon coupling under varying conditions and thus use here parameter sets from recent fits presented in [15] which provides a systematic variation of these four NMPs.

Table I lists the selection of parameter sets and their NMPs. $\mathrm{SV}$-bas is the base point of the variation of forces. Its NMPs are chosen such that dipole polarizability and the three most important giant resonances (GMR, GDR, and GQR) in ${ }^{208} \mathrm{~Pb}$ are well reproduced by Skyrme-RPA calculations. Each one of the next four parameter sets vary exactly one NMP while keeping the other three at the SV-bas value. These $1+4$ parameter sets allow us to explore the effect of each NMP separately. It was figured out in [15] that there is a strong relation between each one of the four NMPs and one specific giant resonance: $K$ affects mainly the GMR, $m^{*} / m$ affects mainly the GQR, $\kappa_{\text {TRK }}$ affects the GDR, and $a_{\text {sym }}$ is linked to the dipole polarizability [34].

TABLE I. Nuclear matter properties for the Skyrme parameter sets used in this study: incompressibility $K$, isoscalar effective mass $m^{*} / m$, symmetry energy $a_{\text {sym }}$, and Thomas-Reiche-Kuhn sum rule enhancement $\kappa_{\mathrm{TRK}}$. The first five parameter sets stem from [15], the last two from [22].

\begin{tabular}{lcccc}
\hline \hline & $K(\mathrm{MeV})$ & $m^{*} / m$ & $a_{\text {sym }}(\mathrm{MeV})$ & $\kappa_{\text {TRK }}$ \\
\hline SV-bas & 234 & 0.90 & 30 & 0.4 \\
SV-kap00 & 234 & 0.90 & 30 & 0.0 \\
SV-mas07 & 234 & 0.70 & 30 & 0.4 \\
SV-sym34 & 234 & 0.90 & 34 & 0.4 \\
SV-K218 & 218 & 0.90 & 30 & 0.4 \\
SV-m64k6 & 241 & 0.64 & 27 & 0.6 \\
SV-m56k6 & 255 & 0.56 & 27 & 0.6 \\
\hline \hline
\end{tabular}

Finally, the last two parameter sets in Table I were developed in [22] with the goal to describe, within TBA, at the same time the GDR in ${ }^{16} \mathrm{O}$ and ${ }^{208} \mathrm{~Pb}$. This required pushing up the RPA peak energy, which was achieved by low $a_{\text {sym }}$ in combination with high $\kappa_{\mathrm{TRK}}$. To avoid unphysical spectral distributions for the GDR, a very low $m^{*} / m$ was used.

\section{The problem of tuning a parameter set}

Looking only at average resonance energies, the tuning of parameter sets is simple. As mentioned before, the three giant resonances which we consider couple each one almost exclusively to one property: the GMR to the incompressibility $K$, the GDR to the TRK sum rule enhancement $\kappa_{\mathrm{TRK}}$, and the GQR to the isoscalar effective mass $m^{*} / m$. This suggests that one can adjust these three resonances independently as needed. However, problems appear when looking at the detailed spectral distributions. We observed in our investigations that the shift in average resonance energies usually does not correspond to a global shift of the spectral distribution, but rather to a redistribution of strength over the spectrum. However, such redistribution can lead to unrealistic profiles.

Figure 1 shows detailed spectra for four parameter sets. SV-kap00 as compared to SV-bas corresponds to a shift of $\kappa_{\mathrm{TRK}}$ from 0.4 (for $\mathrm{SV}$-bas) down to 0 . This has no effect on the GQR

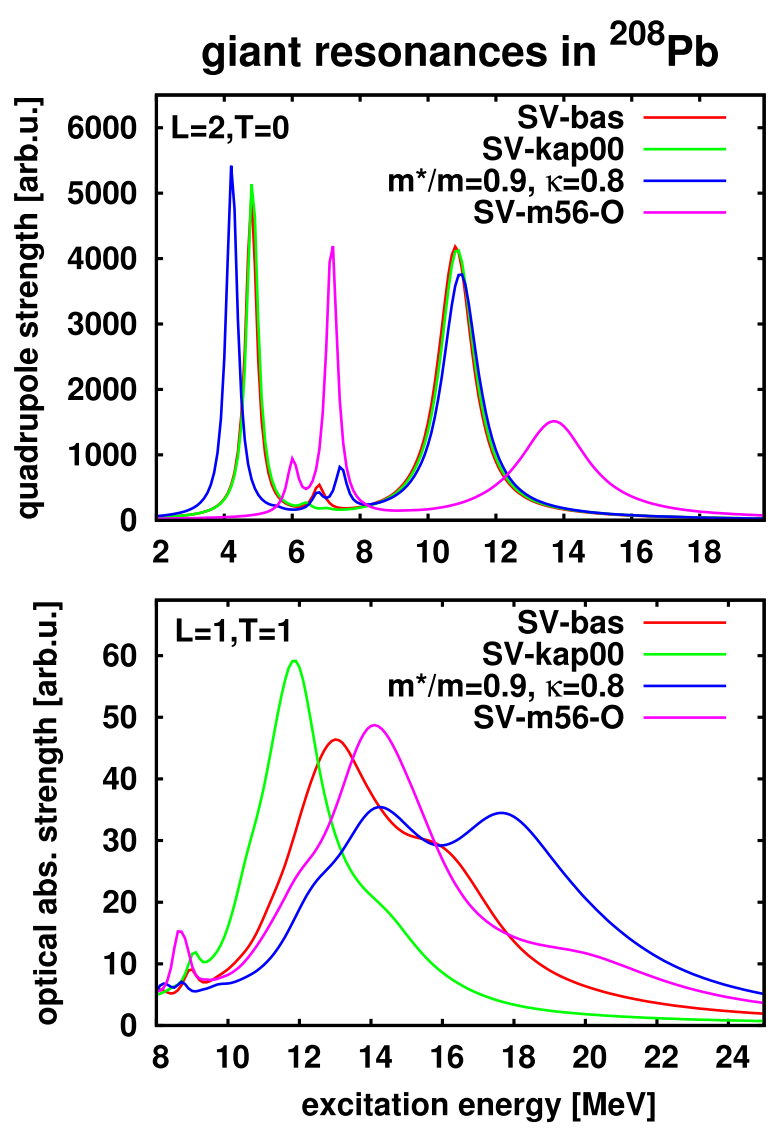

FIG. 1. Dipole strength (lower panel) and quadrupole strength (upper panel) for four parameter sets as indicated. The smooth spectra are obtained from folding with Gaussians of linearly increasing width $\Gamma=\max (0.2,(E-8) / 5) \mathrm{MeV}$. 
and leads to a visible downshift of the GDR. This downshift also changes the profile to the extent that the high-energy bump at $16 \mathrm{MeV}$ in SV-bas now appears at $14 \mathrm{MeV}$ and, more importantly, becomes much smaller. Thus the way from SV-kap00 to SV-bas already changes somewhat the profile, but at a harmless level.

Now we try to up-shift the GDR by enhancing dramatically $\kappa_{\mathrm{TRK}}$ to 0.8 while keeping $m^{*} / m=0.9$ at the value of SV-bas. This leads to the blue curves in the figure. It is gratifying to see that the GQR remains where it should be. The GDR makes the wanted up-shift. However, this happens at the price of a totally unrealistic double humped structure of the GDR. Mind that the upper bump appears in such a pronounced manner in spite of the energy-dependent folding width. Mere enhancement of $\kappa_{\text {TRK }}$ seems thus no solution to the wanted up-shift of the GDR. The former solution was to use much lower $\mathrm{m}^{*} / \mathrm{m}=0.56$ to suppress the double hump. This is successful for the GDR (purple line); however, it is disastrous for the GQR, showing too high GQR position and a grossly unrealistic low-energy spectrum. This is a severe deadlock for global improvement at the level of RPA. The situation becomes more graceful if phonon coupling is included with TBA, as we will see later.

\section{DETAILS OF THE CALCULATION SCHEME}

\section{A. Single-particle basis and residual interaction}

The response functions both for RPA and TBA, Eqs. (9) and (17), are solved in a discrete basis defined as a set of solutions of the Schrödinger equation with box boundary conditions. Both equations are solved in the same large configuration space. A new method to include the continuum in the discrete basis representation is explained in Appendix. The residual interaction $V$ in Eqs. (9) and (17) is derived from the energy functionals according to Eq. (13). In the case of the energy density functional $E[\rho]$ built on the Skyrme forces, the amplitude $V$ determined by Eq. (13) contains zerorange (velocity-independent) and velocity-dependent parts. The scheme for taking into account the zero-range part of the residual interaction adopted in our calculations is described in Refs. [9,13]. A detailed description of the computation of the matrix elements in connection with the Skyrme functional is found in [23].

We will consider only doubly-magic nuclei. They have closed shells and pairing is inactive. The box sizes in the RPA and TBA calculations are $15 \mathrm{fm}$ for ${ }^{16} \mathrm{O},{ }^{40,48} \mathrm{Ca}$ and $18 \mathrm{fm}$ for ${ }^{208} \mathrm{~Pb}$. The single-particle basis in which we solve the RPA and TBA equations includes single-particle states up to $\varepsilon_{\max }=$ $100 \mathrm{MeV}$ (see our discussion in the next two sections). In the TBA calculations we apply the subtraction recipe (18) [31]. As mentioned before, this procedure eliminates double counting, resolves stability problems, and restores the Thouless theorem.

\section{B. Effect of the exact continuum}

The present TBA calculations use a new technique which allows a continuum treatment in connection with full selfconsistency RPA, as outlined in the Appendix. This method uses the discrete basis representation and recovers the exact method [35] of treatment of the continuum in the coordinate

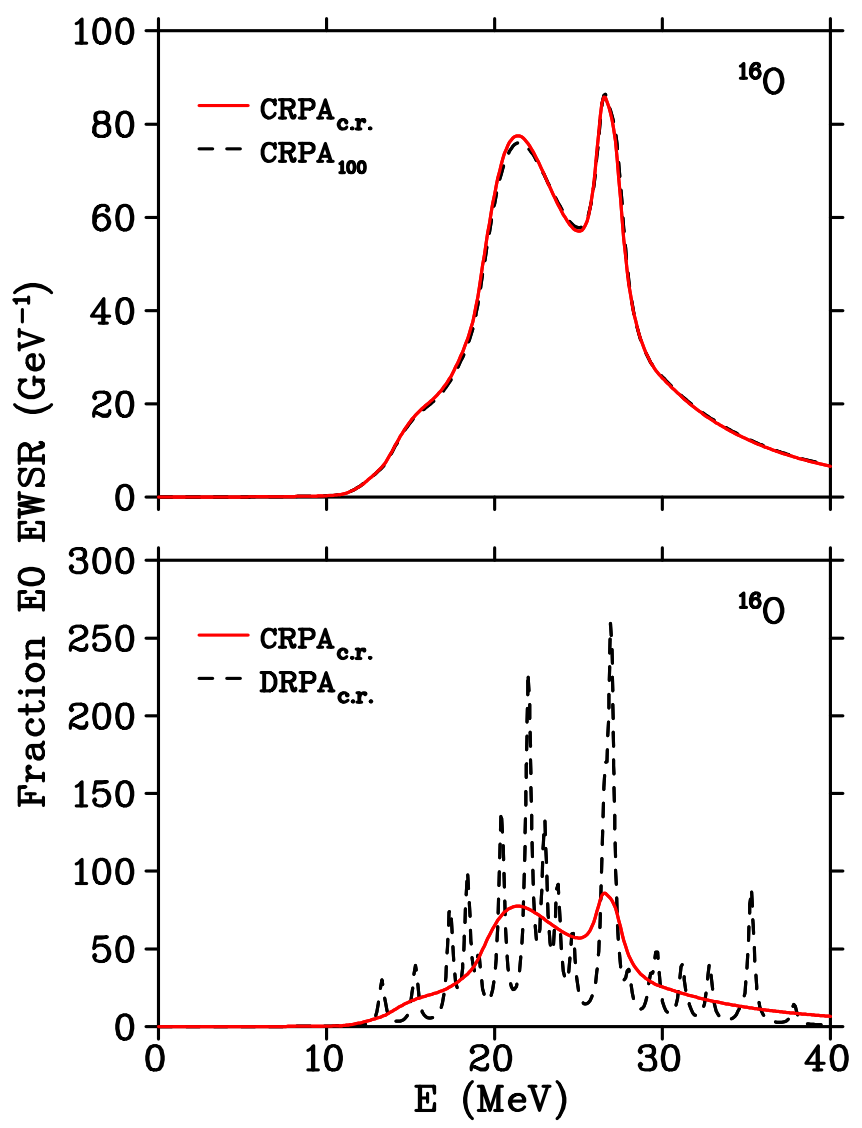

FIG. 2. ISGMR in ${ }^{16} \mathrm{O}$ calculated within fully self-consistent RPA based on the Skyrme energy density functional with the parameter set T6. [36]. Fractions of the E0 EWSR are shown. Upper panel: the $\mathrm{CRPA}_{\text {c.r. }}$ function obtained by making use of the method of Ref. [37] is presented by the red solid line. The CRPA 100 function obtained in the discrete basis with $\varepsilon_{\max }=100 \mathrm{MeV}$ is presented by the black dashed line. Lower panel: the $\mathrm{CRPA}_{\text {c.r. }}$ function (red solid line) is compared with the $\mathrm{DRPA}_{\text {c.r. }}$ function (black dashed line) obtained by the same method as in Ref. [37]. Smearing parameter $\Delta=200 \mathrm{keV}$ was used in all the calculations.

representation if the discrete basis is sufficiently complete ( $\varepsilon_{\max }$ high enough) and the radius of the box $\left(R_{\mathrm{box}}\right)$ is sufficiently large (see the Appendix). To check the accuracy of our method, we first compare the results obtained within the continuum RPA (CRPA) in the discrete basis representation (hereafter called CRPA d.b. $_{\text {. }}$ with the results of the CRPA in the coordinate representation $\left(\mathrm{CRPA}_{\text {c.r. }}\right)$. As an example, we consider calculations of the GMR in the fully self-consistent CRPA based on the Skyrme energy density functional with the parameter set T6 [36] producing the nucleon effective mass $m^{*} / m=1$. As was shown in Ref. [37], the fully self-consistent $\mathrm{CRPA}_{\text {c.r. }}$ scheme in this special case has a relatively simple form. The results for the nucleus ${ }^{16} \mathrm{O}$ are shown in Fig. 2. The function $F(E)$ presented in this figure is the fraction of the energy-weighted sum rule (EWSR) defined as 
where $S(E)$ is the strength function defined in Eq. (5) and $m_{1}=\int d E E S(E)$ is the energy-weighted moment of $S(E)$ determined by the known EWSR [38].

In the upper panel of Fig. 2 the $\mathrm{CRPA}_{\text {c.r. }}$ results are compared with $\mathrm{CRPA}_{100}$ obtained in the discrete basis with $E_{\text {cut }}=100 \mathrm{MeV}$. The details of these CRPA d.b. calculations are the same as in Ref. [10] (that is, the SHF equations were solved in the box with the radius $R_{\text {box }}=15 \mathrm{fm}$ by making use of the Numerov method with the radial mesh size $h=0.05$ fm and with decreasing $h$ at $r<1 \mathrm{fm}$, see Ref. [39]). The equations of the CRPA $\mathrm{A}_{\text {c.r. }}$ were solved in $r$-space with the same mesh spacing $h=0.05 \mathrm{fm}$ and the box size $R_{\mathrm{box}}=15$ fm.

All these calculations used a smearing parameter $\Delta=$ $200 \mathrm{keV}$. The difference between the $\mathrm{CRPA}_{100}$ and $\mathrm{CRPA}_{\text {c.r. }}$ curves is small and hardly visible. The $\mathrm{CRPA}_{300}$ obtained in the discrete basis with $E_{\text {cut }}=300 \mathrm{MeV}$ and $\mathrm{CRPA}_{\text {c.r. }}$ curves are practically indistinguishable, so we do not show them. In the lower panel of Fig. 2 the discrete RPA (DRPA) results obtained by the coordinate representation method of Ref. [37] are compared with the CRPA function for ${ }^{16} \mathrm{O}$ and, again, $\Delta=200 \mathrm{keV}$. In this case, the difference between these results is large.

Thus we see that the magnitude of the continuum effects on nuclear excitations is different in light and heavy nuclei. To see the trend we have calculated the GDR in the nuclei ${ }^{16} \mathrm{O},{ }^{48} \mathrm{Ca}$, ${ }^{132} \mathrm{Sn}$, and ${ }^{208} \mathrm{~Pb}$ within two schemes: $\mathrm{CRPA}_{\text {d.b. }}$ and $\mathrm{DRPA}_{\text {d.b. }}$. using the Skyrme parameter set SV-bas [40]. The results are presented in Fig. 3. In this figure, the photoabsorption cross sections normalized to the classical values $\sigma_{\text {class. }}=\frac{5}{3} \pi\left\langle r^{2}\right\rangle$ are shown. The mean-square radii $\left\langle r^{2}\right\rangle$ have been calculated for each nucleus using its Skyrme-Hartree-Fock ground-state. The $\sigma_{\text {class. }}$ are $378.5 \mathrm{mb}$ for ${ }^{16} \mathrm{O}, 654.5 \mathrm{mb}$ for ${ }^{48} \mathrm{Ca}, 1204.0 \mathrm{mb}$ for ${ }^{132} \mathrm{Sn}$, and $1605.1 \mathrm{mb}$ for ${ }^{208} \mathrm{~Pb}$. As can be seen, the effect of the single-particle continuum is strongest in the light nuclei ${ }^{16} \mathrm{O}$ and ${ }^{40} \mathrm{Ca}$. In the ${ }^{16} \mathrm{O}$ nucleus, the CRPA and DRPA results significantly differ at $\Delta \lesssim 400 \mathrm{keV}$. Even at $\Delta=1 \mathrm{MeV}$ the difference is noticeable. It disappears only at $\Delta=2 \mathrm{MeV}$. In ${ }^{48} \mathrm{Ca}$ the difference between the CRPA and DRPA becomes small at $\Delta \gtrsim 1 \mathrm{MeV}$. The same is true for ${ }^{132} \mathrm{Sn}$, though on the

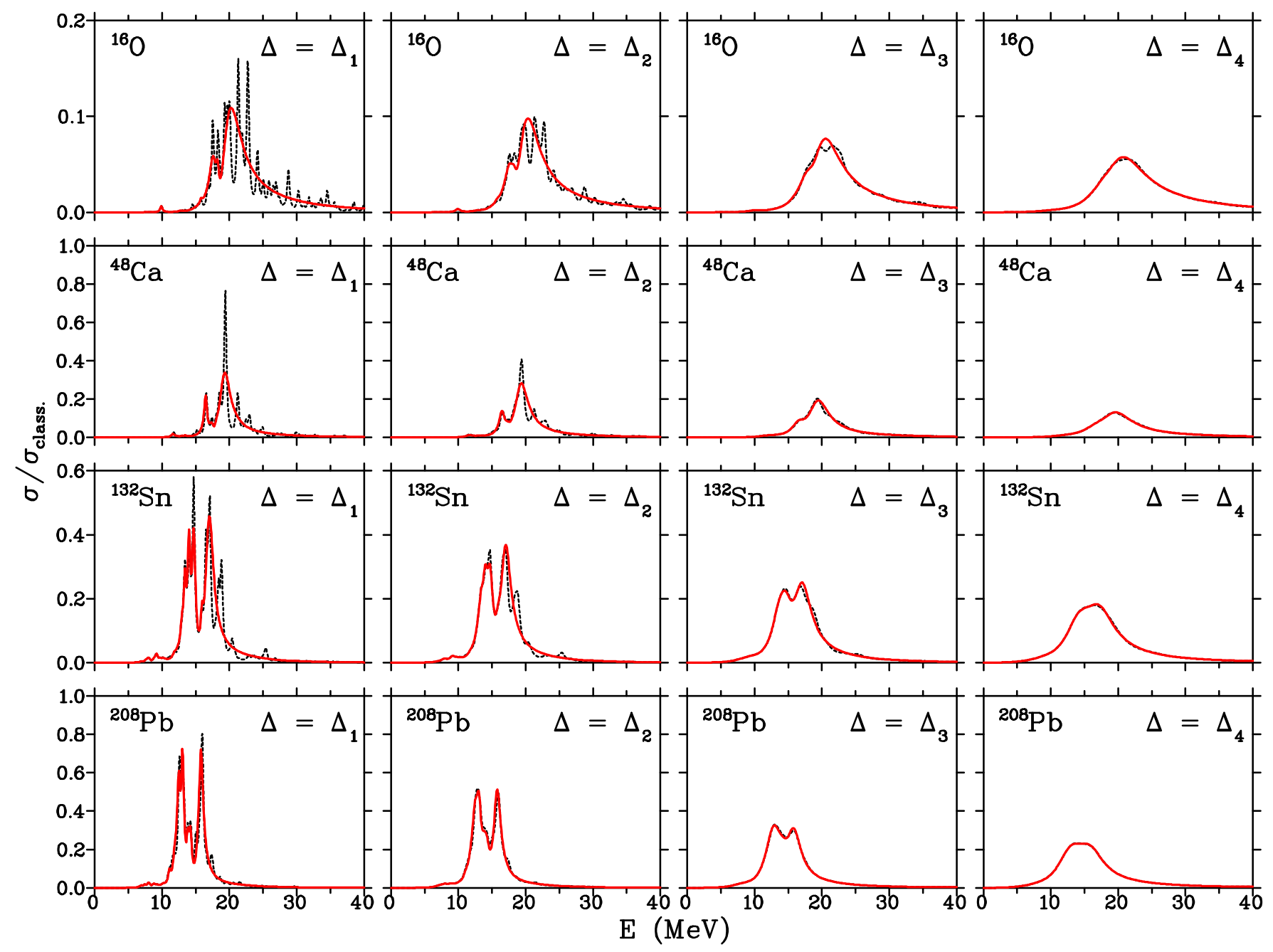

FIG. 3. Photoabsorption cross sections in the nuclei ${ }^{16} \mathrm{O},{ }^{48} \mathrm{Ca},{ }^{132} \mathrm{Sn}$, and ${ }^{208} \mathrm{~Pb}$ calculated within the CRPA (red solid lines) and the DRPA (black dashed lines) with different smearing parameters $\Delta: \Delta_{1}=200 \mathrm{keV}, \Delta_{2}=400 \mathrm{keV}, \Delta_{3}=1 \mathrm{MeV}$, and $\Delta_{4}=2 \mathrm{MeV}$. The discrete basis representation with $E_{\text {cut }}=100 \mathrm{MeV}$ is used both in the CRPA and the DRPA. The calculated cross sections have been normalized to the classical values $\sigma_{\text {class. }}=\frac{5}{3} \pi\left\langle r^{2}\right\rangle$ (see text for more details). The results are obtained with the Skyrme force parameter set SV-bas [40]. 


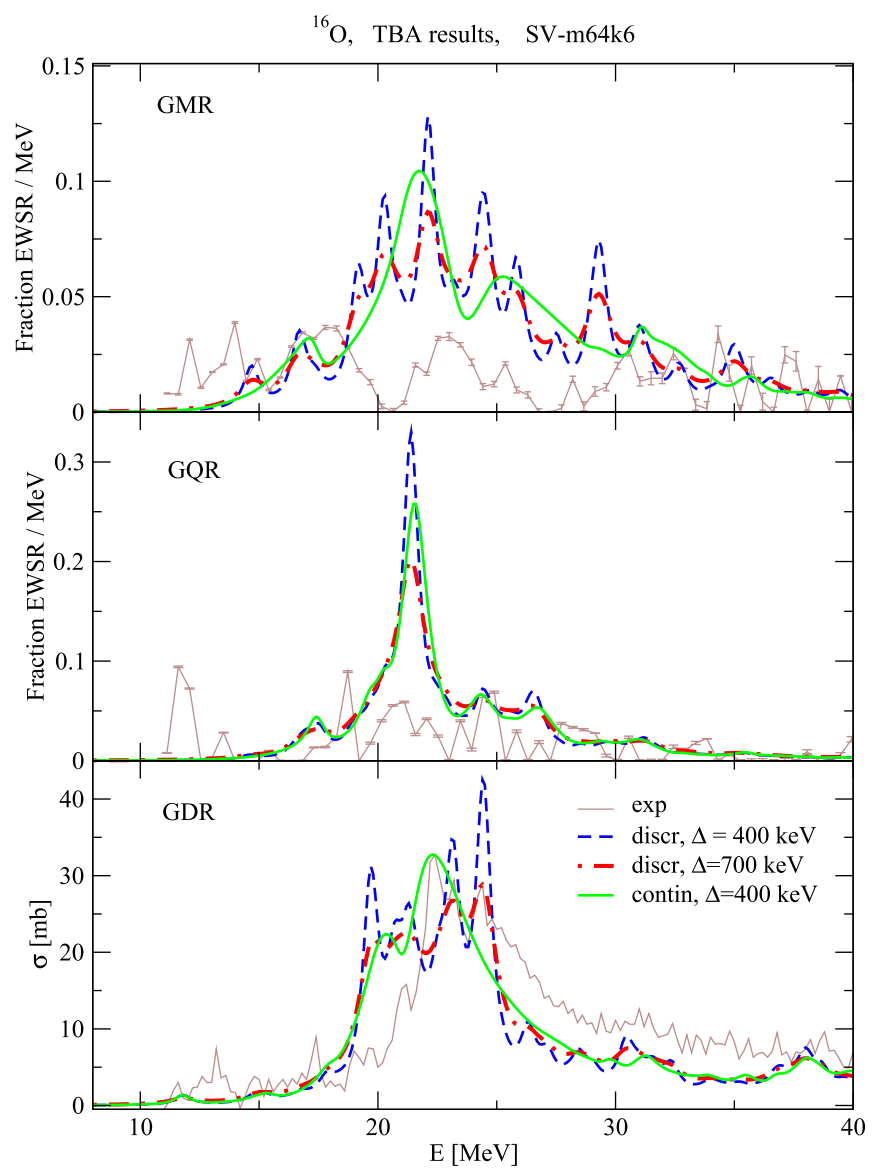

FIG. 4. Discrete and continuum TBA results for ${ }^{16} \mathrm{O}$ which were obtained with the parameter set SV-m64k6. The fractions EWSR for GMR and GQR and photoabsorption cross section for GDR are presented in the upper, middle, and lower panels, respectively. The DTBA for smearing parameters $\Delta=400$ and $700 \mathrm{keV}$ are given by blue dashed and red dashed-doted lines, respectively. Thick green and thin brown full lines represent CTBA with $\Delta=400 \mathrm{keV}$ and experimental data, respectively. The data are taken from Refs. [43,44].

whole this difference here is less than in ${ }^{48} \mathrm{Ca}$. These results are in agreement with the conclusions of Refs. [7,41,42]. In the heavy nucleus ${ }^{208} \mathrm{~Pb}$, the effect of the single-particle continuum is small and is manifested only at $\Delta \lesssim 200 \mathrm{keV}$.

In Fig. 4, for ${ }^{16} \mathrm{O}$, and Fig. 5 , for ${ }^{40} \mathrm{Ca}$, we compare the TBA results obtained with the exact continuum treatment (CTBA) and the discretized approximation (DTBA). Here, blue dashed and red dashed-dotted lines represent the DTBA results for smearing parameters $\Delta=400$ and $700 \mathrm{keV}$, respectively. The expression "strength" in the $Y$ axes mean fractions EWSR for GMR and GQR and photoabsorption cross sections for GDR. The experimental data for GMR and GQR in ${ }^{16} \mathrm{O}$ were taken from Ref. [43] and for GDR in ${ }^{16} \mathrm{O}$ from [44]. The data for ${ }^{40} \mathrm{Ca}$ were taken from Refs. [45] and [46], respectively. The figures show that, for light nuclei, increasing $\Delta$ (DTBA) damps the artificial fine structure of the discrete approach. But, at the same time, it wipes out important physical features. Hence, it is impossible to reproduce CTBA results for strength functions of light nuclei by using the DTBA, both with small and large smearing parameters.

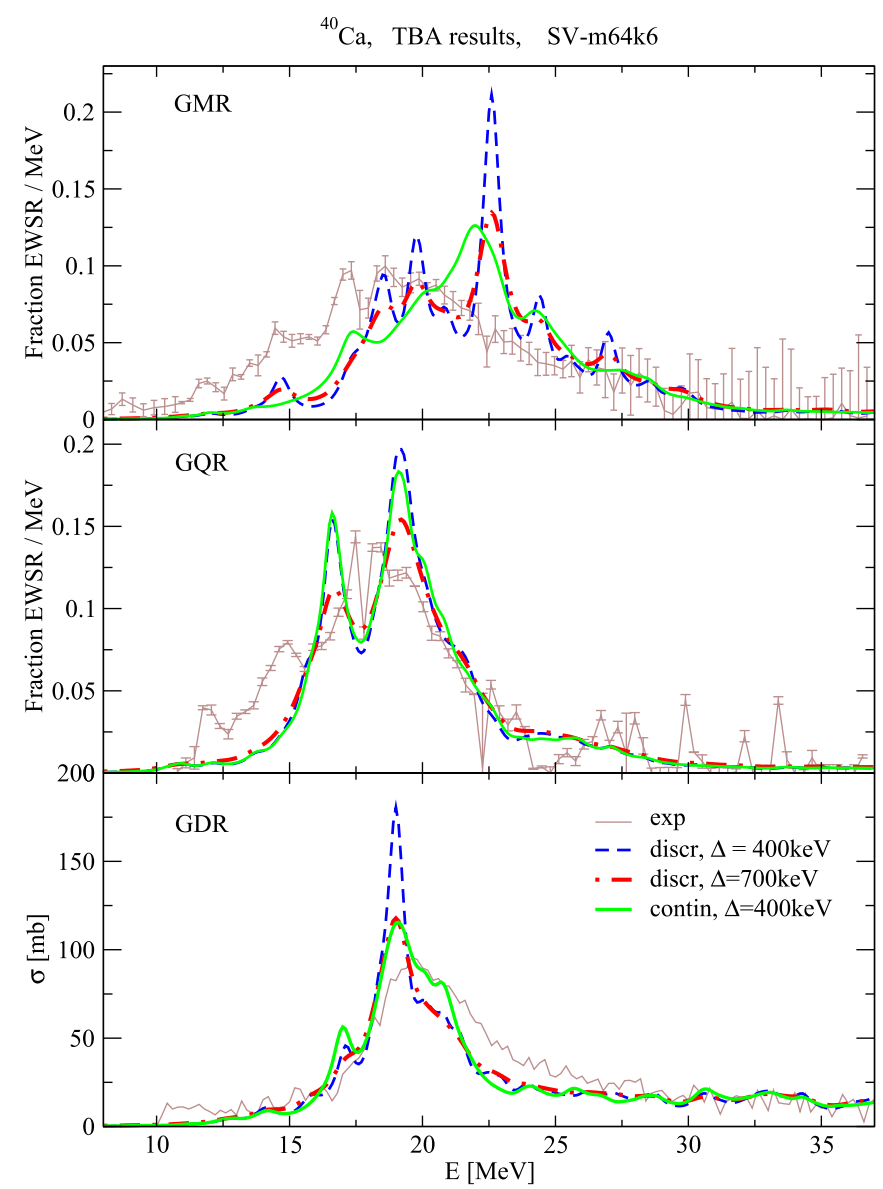

FIG. 5. Same as in Fig. 4 but for ${ }^{40} \mathrm{Ca}$. The corresponding data are taken from Refs. [45,46].

The experimental profiles for the two isoscalar resonances in ${ }^{16} \mathrm{O}$ look very different from the isovector GDR and from all resonances in heavier nuclei. The theoretical GQR shows a narrow peak whereas the experimental strength is nearly continuously distributed over more than $20 \mathrm{MeV}$. The same is true also for the experimental GMR strength. Here the theoretical strength distribution is very broad and shows at least some qualitative similarity. There are little differences between the various parameter sets. The question arises, why are we not able to reproduce theoretically these two resonances while the results in the heavier nuclei are in good qualitative in many cases even in quantitative agreement with experiment? For the GQR the explanation is simple: The dominant decay channel of the GQR in ${ }^{16} \mathrm{O}$ is the $\alpha$ decay into the ground state and the first excited state of ${ }^{12} \mathrm{C}$ [47]. In the range $18-23 \mathrm{MeV}$ the $\alpha$-decay width is $90 \%$ of the total decay width and in $23-27 \mathrm{MeV}$ it is $70 \%$. This reaction mechanism is included in neither RPA nor TBA. This is probably the reason why theory overestimates the peak height of the cross section and does not reproduce the very broad experimental distribution. While the theoretical GQR cross section in ${ }^{16} \mathrm{O}$ shows a well concentrated resonance, the theoretical monopole distribution is very broad, as no narrow single-particle resonances can contribute. It resembles more the experimental pattern but is at least a factor of 2 too high in the resonance region. The situation is completely different 
for the GDR. Our continuum calculation reproduces nearly quantitatively the shape and magnitude of the experimental distribution. The reason is that the GDR is dominated by the $1 \hbar \omega$ transitions which practically exhaust the TRK sum rule. However, the peaks of the distribution are typically $1 \mathrm{MeV}$ too low for the present Skyrme parameter set.

Figure 5 compares DTBA and CTBA for the case of ${ }^{40} \mathrm{Ca}$. The agreement between theory and experiment is very good for the GQR and GDR. In the case of the GMR our theoretical distribution is about $2 \mathrm{MeV}$ too high compared with the experimental distribution.

It should be emphasized that all our results, except for those cases which are explicitly stipulated otherwise, were obtained with the exact treatment of the $s p$ continuum. Therefore, in most of the cases, we use the simple abbreviations RPA and TBA instead of CRPA and CTBA.

\section{The dependence on the number of phonons and on the size of the single-particle basis in the complex configurations}

In all the TBA calculations we use a large single-particle (s.p.) basis both in the phonons and in the complex (1p-1h $\otimes$ phonon) configurations; that is, a large number of $1 \mathrm{p}-1 \mathrm{~h}$ states in these configurations. As it was mentioned in Sec. III A, the upper limit for s.p. energies in all calculations for all nuclei was $\varepsilon_{\max }=100 \mathrm{MeV}$. At the same time, only collective phonons were used in the complex configurations.
The dependence of the theoretical results on the number of phonons used in the calculation is of crucial importance. For this reason we investigate this question in some detail. The result of our investigations for the GDR in ${ }^{16} \mathrm{O}$ is summarized in Fig. 6. The energies $E_{0}$ and the widths $\Gamma$ where derived from the theoretical cross section by a Lorentzian fit. We performed TBA calculations with and without the subtraction procedure. The two approaches give very different results. For comparison the RPA results are shown in the left upper corner of each figure.

In the left column of Fig. 6, the dependence of $E_{0}$ and $\Gamma$ is presented as a function of the maximal phonon energies $E_{\max }^{\text {phon }}$. From Table II, one obtains the connection between $E_{\max }^{\text {phon }}$ and the number of phonons considered in each calculation. The single-particle basis in which we solve the RPA and TBA equations includes s.p. states up to $\varepsilon_{\max }=100 \mathrm{MeV}$ with angular momenta up to $L_{\max }=17$. In the right column the same quantities are shown as a function of the lower cutoff for transition strength $B_{\text {cut }}$ of the phonons, where

$$
B_{\mathrm{cut}}=B(E L) / B(E L)_{\max }
$$

$B(E L)_{\max }$ is the maximal reduced probability of the excitation of the phonon states with the given angular momentum $L$. The connection between $B_{\text {cut }}$ and the number of phonons can be found again in Table II. A too large number of phonons causes two problems: violation of the Pauli principle and double counting. We reduce these problems as we restrict ourselves in
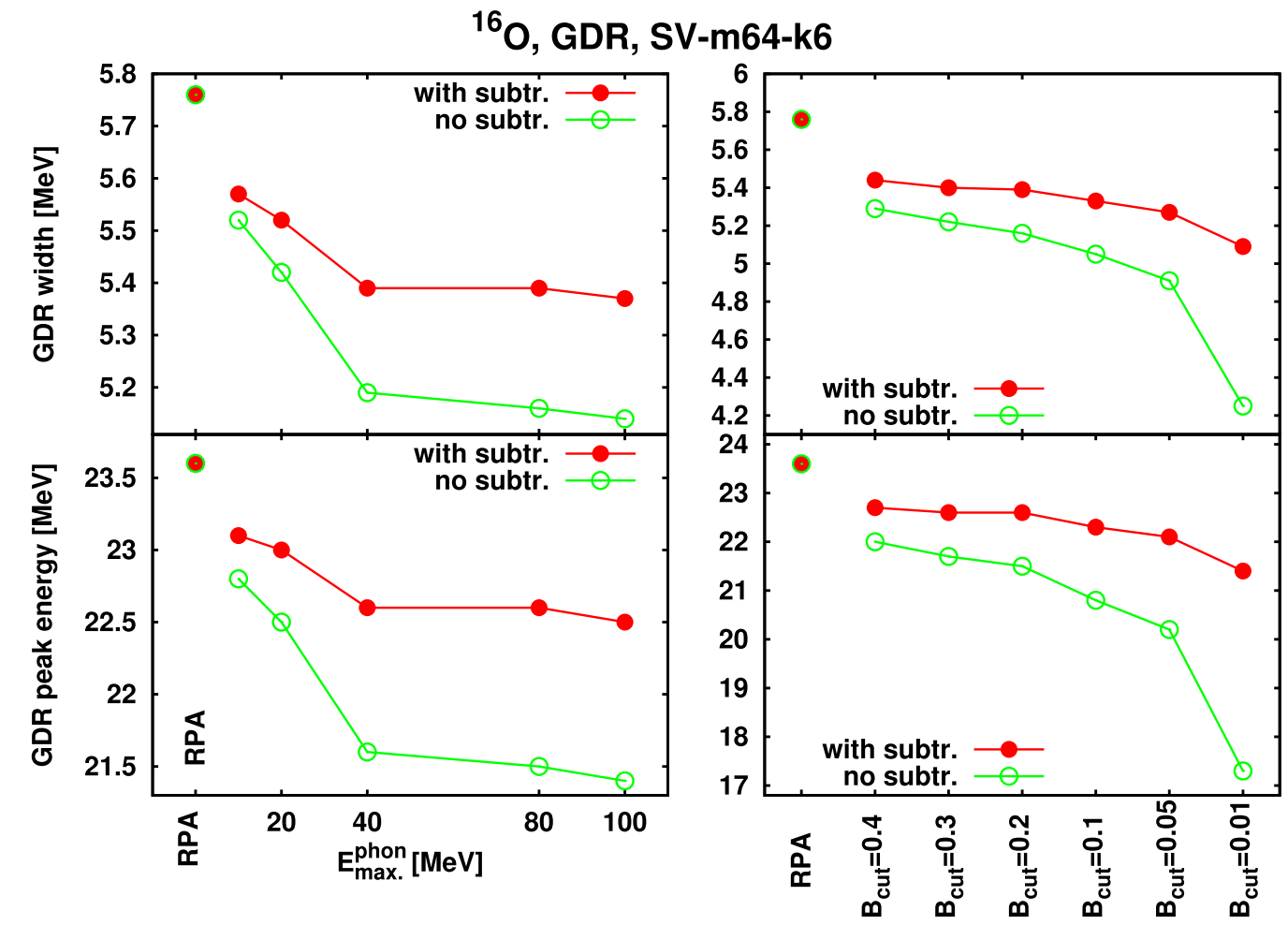

FIG. 6. Energy (lower part) and width (upper part) of the GDR in ${ }^{16} \mathrm{O}$ obtained from TBA calculations. The energy $E_{0}$ and the width $\Gamma$ are the corresponding parameters of a Lorentzian fit to the theoretical results. In the left corner of each figure the RPA result is given. In the left column we present $E_{0}$ and $\Gamma$ as a function of the maximal phonon energy $E_{\max }^{\text {phon }}$ used in complex configurations. In the right column we present $E_{0}$ and $\Gamma$ as function of the minimal collectivity $B_{\text {cut }}$ of the phonons but with fixed value $E_{\max }^{\text {phon }}=80 \mathrm{MeV}$. Table II gives the relation between values $E_{\max }^{\text {phon }}, B_{\text {cut }}$, and the number of phonons. 
TABLE II. Relation between $E_{\max }^{\text {phon }}$ and the number of phonons used in $1 \mathrm{p}-1 \mathrm{~h} \otimes$ phonon configurations for ${ }^{16} \mathrm{O}$. The force SV-m64k6 was used. The phonons were obtained in the $s p$ basis with $\varepsilon_{\max }=$ $100 \mathrm{MeV}$ and angular momenta up to $L_{\max }=17$. Only collective phonons were used in our actual TBA calculations; that is, phonons with $B_{\text {cut }} \equiv B(E L) / B(E L)_{\max } \geqslant 0.2$. (see also the text). Under these conditions, the number of phonons is fixed by the maximum phonon energy $E_{\max }^{\text {phon }}$. The effect of the noncollective phonons is demonstrated for small values $B_{\text {cut }}$ in the last two columns.

\begin{tabular}{lcccccccccc}
\hline \hline$B_{\text {cut }}$ & 0.4 & 0.3 & 0.2 & 0.2 & 0.2 & 0.2 & 0.2 & 0.1 & 0.05 & 0.01 \\
$E_{\text {max }}^{\text {phon }}$ & 80 & 80 & 10 & 20 & 40 & 80 & 100 & 80 & 80 & 80 \\
$N_{\text {phon }}$ & 42 & 52 & 1 & 6 & 55 & 66 & 69 & 117 & 166 & 325 \\
\hline \hline
\end{tabular}

the actual calculations on phonons with $B_{\text {cut }} \geqslant 0.2$. Between $E_{\text {max }}^{\text {phon }}=40 \mathrm{MeV}$ and $E_{\max }^{\text {phon }}=80 \mathrm{MeV}$ the energy and width remain stable if one applies the subtraction procedure. This corresponds to 55 phonons and 66 phonons, respectively (see Table II and the text). In the right column the effect of an even larger number of phonons is presented. Here the transition strength parameter $B_{\text {cut }}$ ranges from 0.4 down to 0.01 . Here one sees strong changes only for the extreme cases of $B_{\text {cut }}=0.05$ and 0.01. Note that the decrease of the GDR width in the TBA as compared to the RPA shown in Fig. 6 is explained by the different energy shifts of the RPA states in ${ }^{16} \mathrm{O}$, which is a consequence of the small number of low-energy $2 \mathrm{p}-2 \mathrm{~h}$ configurations in this nucleus. The large spreading widths in other nuclei (e.g., in ${ }^{208} \mathrm{~Pb}$ ) lead to the opposite effect.
TABLE III. Dependence of the resonance energy and width (Lorentzian parameters $E_{0}$ and $\Gamma$ ) on the size of the sp basis used in phonons and in $1 \mathrm{p}-1 \mathrm{~h} \otimes$ phonon configurations for ${ }^{208} \mathrm{~Pb}$ with $B_{\text {cut }}=$ 0.2 . The size of the basis is characterized by the maximum energy $\varepsilon_{\max }$. For the GDR, the calculated parameters were photoabsorption cross sections while for GMR and GQR the fractions EWSR were used. The force SV-m64k6 was used. All the values are given in MeV.

\begin{tabular}{lccccccc}
\hline \hline$\varepsilon_{\max }$ & \multicolumn{3}{c}{50} & \multicolumn{2}{c}{100} & \multicolumn{2}{c}{150} \\
$E_{\max }^{\text {phon }}$ & \multirow{2}{*}{ RPA } & \multicolumn{2}{c}{40} & \multicolumn{2}{c}{40} & \multicolumn{2}{c}{40} \\
subtract. & & no & yes & no & yes & no & yes \\
\hline GDR $E_{0}$ & 15.0 & 13.5 & 14.4 & 13.3 & 14.3 & 13.3 & 14.3 \\
$\Gamma$ & 4.60 & 4.63 & 4.57 & 4.61 & 4.53 & 4.63 & 4.54 \\
GMR $E_{0}$ & 14.4 & 13.3 & 14.1 & 13.1 & 14.0 & 13.0 & 13.9 \\
$\Gamma$ & 1.53 & 2.09 & 2.15 & 2.08 & 2.18 & 2.04 & 2.14 \\
GQR $E_{0}$ & 12.8 & 11.1 & 11.9 & 10.9 & 11.8 & 10.8 & 11.7 \\
$\Gamma$ & 1.04 & 1.10 & 1.13 & 1.10 & 1.19 & 1.10 & 1.24 \\
\hline \hline
\end{tabular}

The same is true also for the isoscalar resonances GMR and GQR, as can be seen in Fig. 7. From this investigation we conclude that our results in ${ }^{16} \mathrm{O}$ are stable for $\varepsilon_{\max }=100 \mathrm{MeV}$ and 55 phonons.

In Table III we compare again TBA results obtained with and without the subtraction procedure as a function of the s.p. space. Here we used $B_{\text {cut }}=0.2$ which corresponds to 40 phonons. The results where the subtraction method was applied are very stable.

${ }^{16} \mathrm{O}$, RPA and TBA, isoscalar $\mathrm{L}=0+\mathrm{L}=2$, force=SV-m64-k6

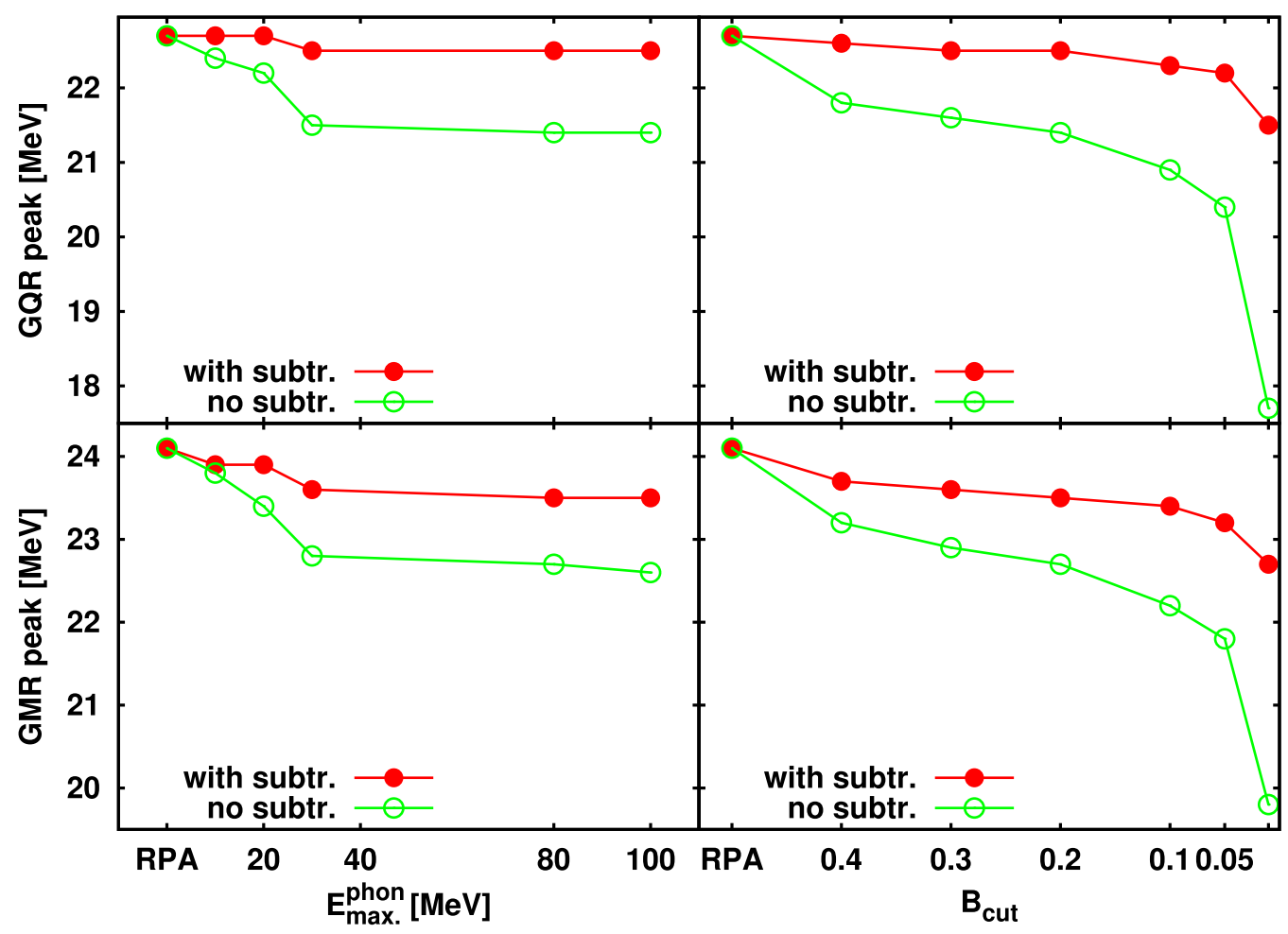

FIG. 7. Same as in Fig. 6 but for the GMR and GQR in ${ }^{16} \mathrm{O}$. 


\section{RESULTS}

We concentrate on the three most important giant resonances: the isoscalar giant monopole resonance (GMR), the isoscalar giant quadrupole resonance (GQR), and the isovector giant dipole resonance (GDR).

\section{A. Comparison with experiment}

In Fig. 8, the theoretical cross sections of the GMR, GQR, and GDR are compared with the experimental ones for ${ }^{208} \mathrm{~Pb}$. The theoretical results are calculated with the seven Skyrme parameter sets which we presented in Table I of Sec. II B.

The peak energy of the GDR is expected at $80 A^{-\frac{1}{3}}=$ $14 \mathrm{MeV}$ in ${ }^{208} \mathrm{~Pb}[14]$. The experimental photoabsorption strength shows a Lorentzian-like distribution with only minor fluctuations [48]. The RPA is not able to reproduce the experimental strength distribution. There are two major peaks for all seven Skyrme parameter sets used. The centroid energies of both peaks depend sensitively on the specific parameter set employed. Since we include the nuclear continuum, discretization effects are ruled out as a possible explanation of the unrealistic double humped structure. An energy dissipation mechanism other than the RPA is needed. The phonon coupling provides such a mechanism. The TBA produces a strength distribution with only one major peak for all parameter sets employed. The quantitative agreement with the experimental strength is reasonably good for five of the seven parameter sets, while the sets SV-kap00 and SV-sym34 perform less well.
${ }^{208} \mathrm{~Pb} \quad \mathrm{~L}=0, \mathrm{~T}=0$ fraction EWSR $/ \mathrm{MeV}$
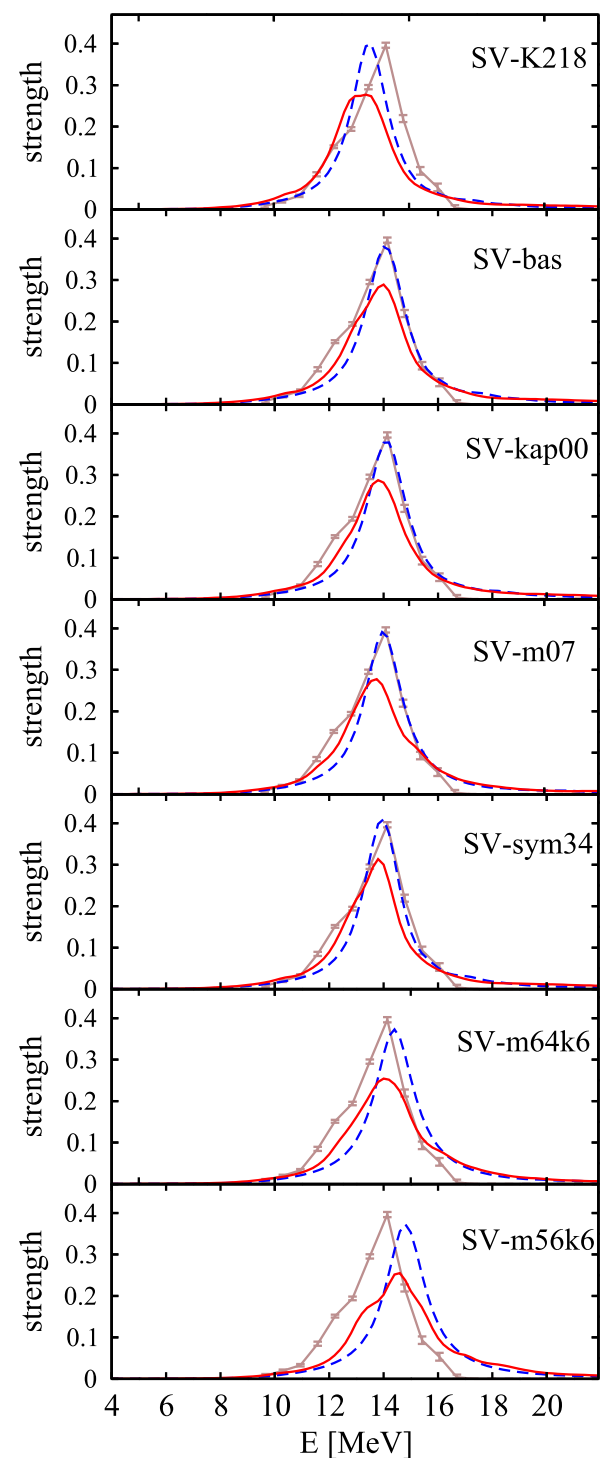

${ }^{208} \mathrm{~Pb} \quad \mathrm{~L}=2, \mathrm{~T}=0$ fraction EWSR / MeV

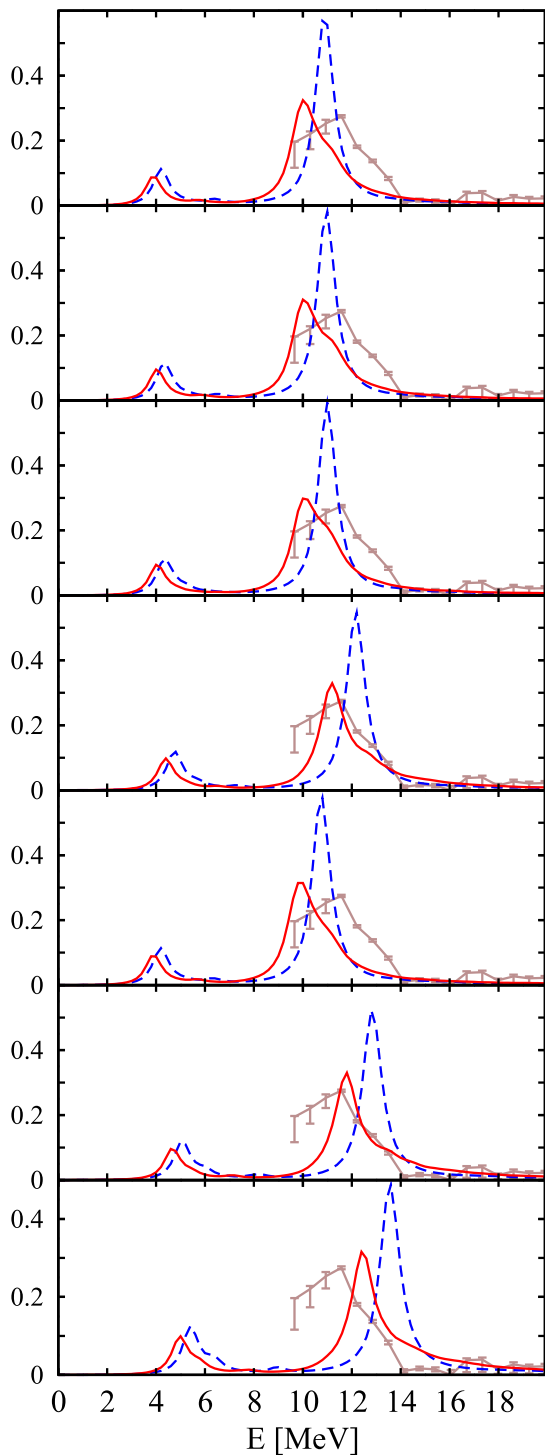

${ }^{208} \mathrm{~Pb} \quad \mathrm{~L}=1, \mathrm{~T}=1 \quad \sigma[\mathrm{mb}]$

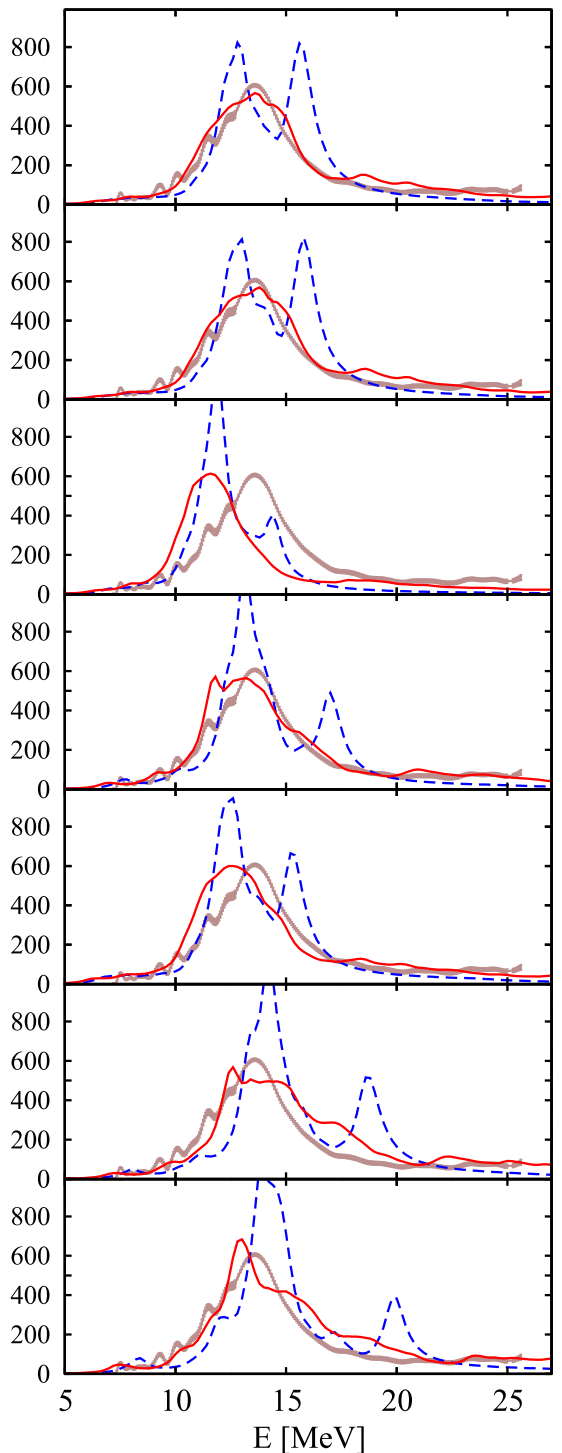

FIG. 8. Spectral strength distributions for ${ }^{208} \mathrm{~Pb}$ and the three modes under consideration: isoscalar monopole (left panels), isoscalar quadrupole (middle panels), and isovector dipole (right panels). Photoabsorption strength is shown in case of the dipole mode. Results are obtained with the seven Skyrme parameter sets discussed in Sec. II B. Compared are strengths derived from RPA (blue dashed) and TBA (full red) with experimental data (full brown) from [48] for the GDR and [49] for the GMR and the GQR. 
${ }^{48} \mathrm{Ca} \quad \mathrm{L}=0, \mathrm{~T}=0$ fraction $\mathrm{EWSR} / \mathrm{MeV}$

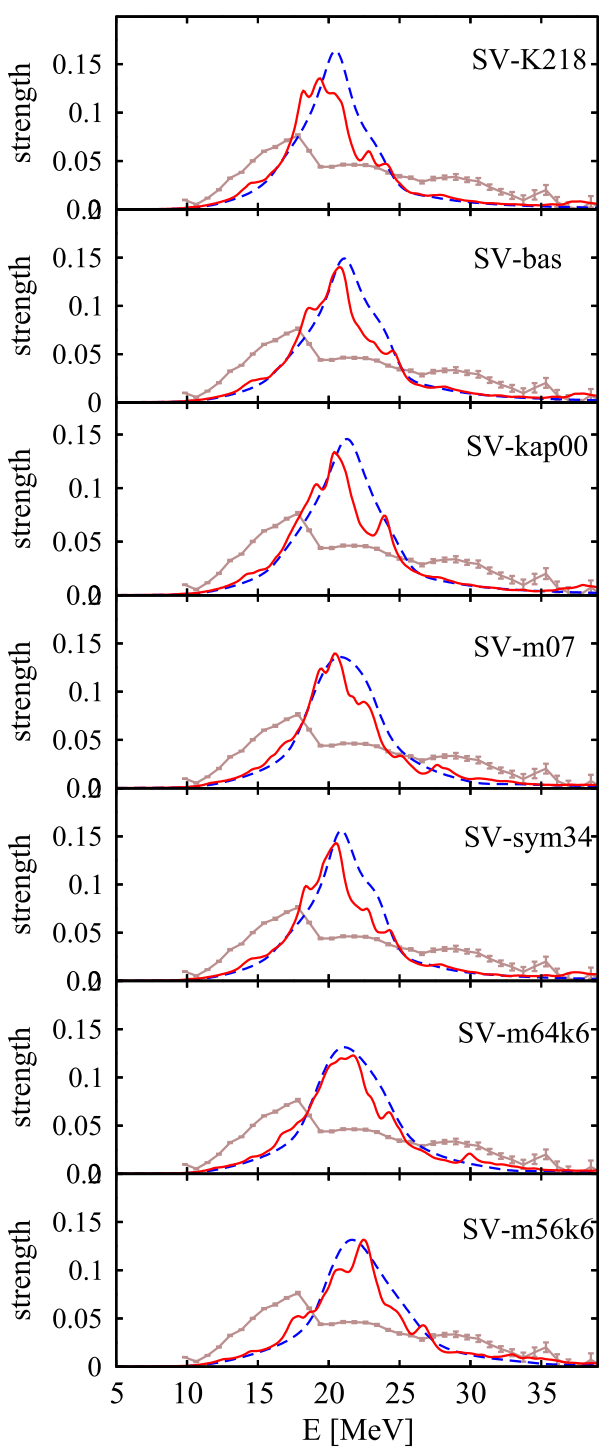

${ }^{48} \mathrm{Ca} \quad \mathrm{L}=2, \mathrm{~T}=0$ fraction EWSR $/ \mathrm{MeV}$

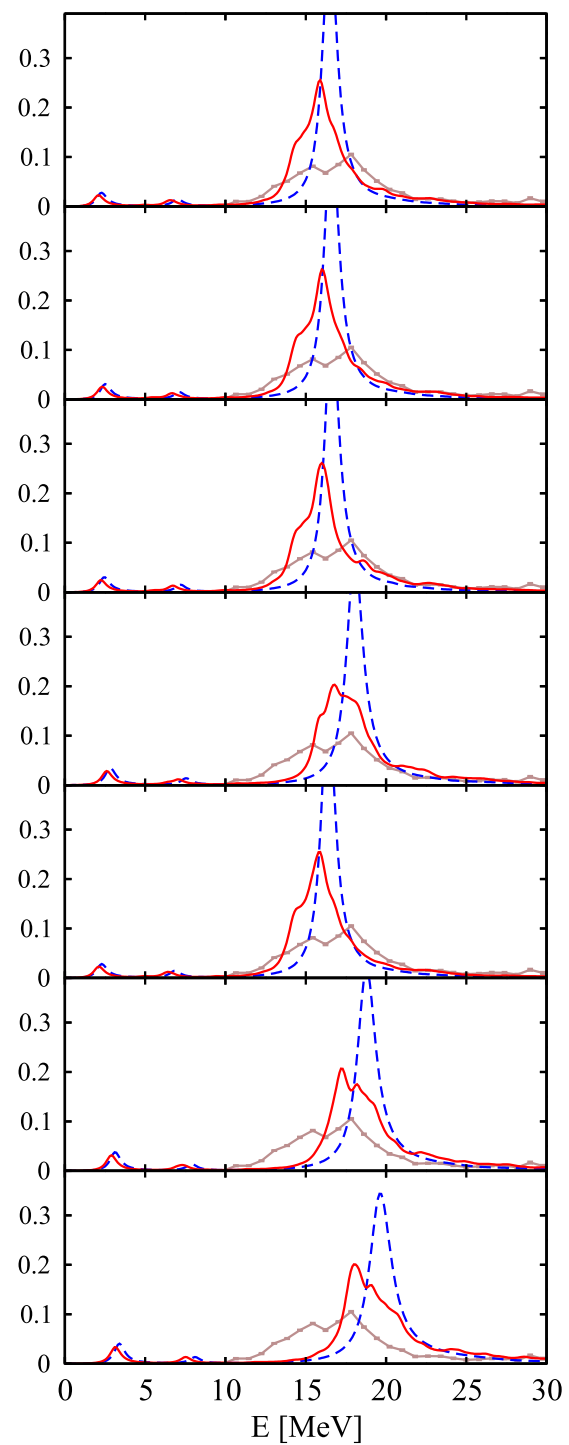

${ }^{48} \mathrm{Ca} \quad \mathrm{L}=1, \mathrm{~T}=1 \quad \sigma[\mathrm{mb}]$

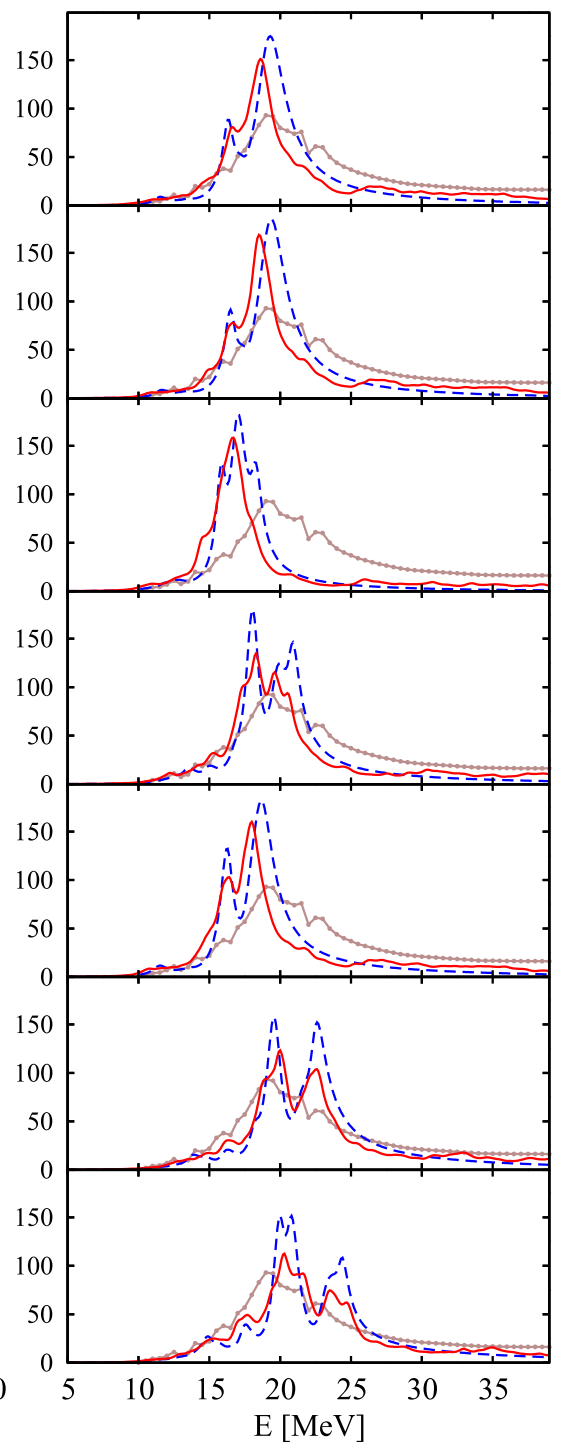

FIG. 9. Same results as in the previous figure but for ${ }^{48} \mathrm{Ca}$. The data are taken from [46] for the GDR and from [45] for the GMR and the GQR.

The experimental GQR strength is centered near $12 \mathrm{MeV}$. The RPA produces a peak in the vicinity of the experimental one, but overestimates the experimental peak height by approximately a factor 2 , while simultaneously underestimating the width. The TBA introduces a larger width and thus leads to an improved description of the data.

The experimental peak energy of the GMR is reproduced both by the RPA and the TBA, while the peak height is reduced by the TBA.

In Fig. 9 we compare our theoretical results for ${ }^{48} \mathrm{Ca}$ with the data.

The experimental GDR strength in ${ }^{48} \mathrm{Ca}$ shows a Lorentzlike distribution with one peak at approximately $18 \mathrm{MeV}$. RPA yields consistently a too high and too narrow peak as compared to the experimental strength, and shows up to two major peaks. The sets SV-kap00 and SV-sym34 generate the largest discrepancies in comparison with data, as was also observed in ${ }^{208} \mathrm{~Pb}$. The other five parameter sets reproduce the energy of the experimental peak reasonably well, but overestimate the strength. Including the phonon coupling within the TBA, a reduction of the peak height is obtained, but the TBA result still overestimates the data. The sets SV-m64k6 and SV-m56k6 which were fine-tuned [22] to reproduce the GDR in ${ }^{208} \mathrm{~Pb}$ and ${ }^{16} \mathrm{O}$ show a fair agreement with the data.

The experimental GQR and GMR strengths are very broad so that it is not obvious how to identify a peak energy. The RPA produces one narrow resonance, in contrast to the data. The TBA is able to reduce the GQR peak height, but still overestimates the experimental strength, while for the GMR strength the differences between RPA and TBA are small.

One may understand the present results as a hint that there are additional energy dissipation mechanisms other than phonon coupling which are relevant in light nuclei. The experimental fact that the GQR in ${ }^{16} \mathrm{O}$ decays mainly via $\alpha$ 
emission suggests a possible reaction channel not included in the present approach. At present, it is not known how to incorporate such complex reaction mechanisms into the present framework.

\section{B. Analysis of the results}

In order to analyze the effect of the phonon degree of freedom on the results, we characterize each resonance by one number, the energy centroid, defined as the ratio $m_{1} / m_{0}$ of the first and zeroth energy moment of the corresponding strengths. The moments are collected in exactly the same energy windows which were used in the experiments. These windows are $11<E<40 \mathrm{MeV}$ for GMR and GQR in ${ }^{16} \mathrm{O}$, $15<E<30 \mathrm{MeV}$ for the GDR in ${ }^{16} \mathrm{O}, 10<E<30 \mathrm{MeV}$ for GMR in ${ }^{40,48} \mathrm{Ca}$, and $10<E<25 \mathrm{MeV}$ for $\mathrm{GQR}$ in ${ }^{40,48} \mathrm{Ca}$, The centroids $E_{0}$ for the GDR in ${ }^{40,48} \mathrm{Ca}$ and for the GDR, GMR, and GQR in ${ }^{208} \mathrm{~Pb}$ were calculated in the window $E_{0} \pm 2 \delta$, where $\delta$ is the spectral dispersion (although with constraint $\delta \geqslant 2 \mathrm{MeV}$ ).

Figure 10 summarizes the centroids for the three major giant resonances in ${ }^{208} \mathrm{~Pb}$ (upper and middle) and the dipole polarizability $\alpha_{D}$ (lower panel). Let us briefly recall the trends for RPA. Changing $\kappa_{\mathrm{TRK}}$ affects almost exclusively the GDR such that lower $\kappa_{\mathrm{TRK}}$ yields a lower peak position. Changing $m^{*} / m$ affects the GQR where lower $m^{*} / m$ means higher peak position. Changing $a_{\text {sym }}$ affects the dipole polarizability $\alpha_{D}$ with larger $a_{\text {sym }}$ enhancing $\alpha_{D}$, although we see also a small side effect on $\alpha_{D}$ from changing $m^{*} / m$. Changing $K$ has an impact predominantly on the GMR, where lower $K$ lowers the peak energy. The combined changes of NMPs in the two parameter sets SV-m64k6 and SV-m56k6 yield changes in every mode.

The effect of the phonon coupling (moving from open to closed symbols) does not change these trends in general. The effects in detail depend very much on the actual parameter set, but in all cases the energies are shifted downwards. The lower panel of Fig. 10 shows the dipole polarizability $\alpha_{D}$. The polarizability represents a static response, and TBA by virtue of the subtraction method is designed such that it leaves stationary states unchanged. Thus RPA and TBA results for $\alpha_{D}$ are exactly the same, which simplifies discussions in this case. The large deviation of $\alpha_{D}$ for SV-sym34 is the obvious effect of $a_{\text {sym }}$. It is noteworthy that the combination of changes to NMPs in SV-m56k6 cooperate to achieve a good description of $\alpha_{D}$. Here, the low $a_{\text {sym }}$ alone would have produced a too low $\alpha_{D}$. But the low $m^{*} / m$ drives $\alpha_{D}$ back up again.

Figure 11 shows the same for the light nucleus ${ }^{16} \mathrm{O}$. It is well known that the standard Skyrme forces produce all too low GDR energies (second panel from below) while those with exotically low effective mass (SV-m56k6 and SV-m64k6) perform fine. The situation is exactly opposite for the GQR (upper panel). Here the standard forces do well and the exotic ones fail. The GMR is badly reproduced. All forces yield a too high centroid energy. As the GMR and GQR are nearly continuously distributed, the definition of a centroid energy and a width depends strongly on the integration intervals chosen and is used here only as a simple tool to facilitate the discussion. To summarize the situation one may conclude

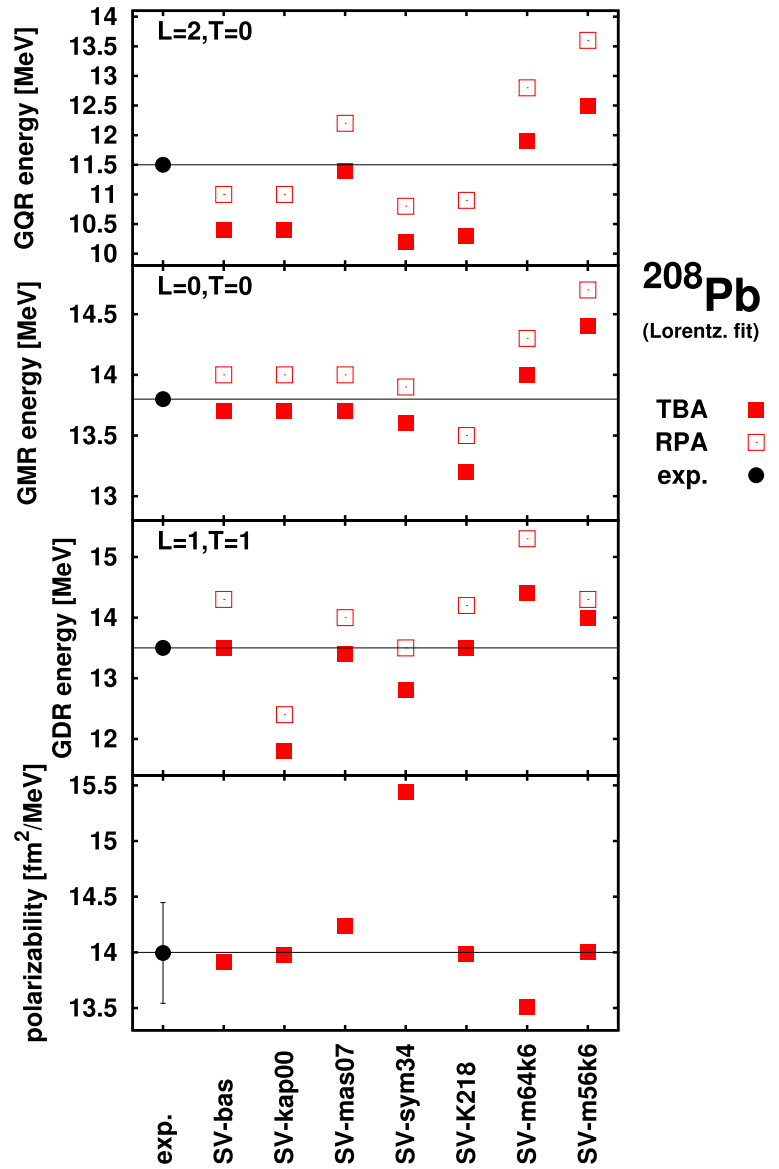

FIG. 10. Comparison of giant resonance energies in ${ }^{208} \mathrm{~Pb}$ for a variety of Skyrme parameter sets as indicated. The energy centroids $E_{0}=m_{1} / m_{0}$ are computed in the window $E_{0} \pm 2 \delta$, where $\delta$ is a dispersion (with the condition $\delta \geqslant 2 \mathrm{MeV}$ ). Open and filled symbols show the values calculated in the frameworks of RPA and TBA, respectively. The experimental data are taken from Refs. [48] for the GDR, [49] for the GMR, and the GQR, and [19] for $\alpha_{D}$.

as follows: For ${ }^{208} \mathrm{~Pb}$ alone, the conventional RPA using the parameter set SV-bas manages to provide a good description for all four features. However, SV-bas fails badly for the GDR in ${ }^{16} \mathrm{O}$ and to some extent also for the polarizability (the mismatch of GMR is ignored here). It is only the new force SV-m56k6 in combination with TBA which manages to get the GDR correct in both nuclei [22]. But this spoils GMR, GQR, and $\alpha_{D}\left({ }^{16} \mathrm{O}\right)$. Considering the whole synopsis, we realize that there is no force which reproduces all three giant resonances and the polarizability simultaneously in ${ }^{16} \mathrm{O}$ and ${ }^{208} \mathrm{~Pb}$, for either RPA or TBA.

Figure 12 shows the differences of the energy centroids between TBA and RPA for ${ }^{208} \mathrm{~Pb}$ and ${ }^{16} \mathrm{O}$. In all cases the TBA energies are lower than the RPA results. This is probably due to the first-order correction in the energy dependence of the effective mass discussed in Sec. II A. The shifts are between $1 \mathrm{MeV}$ for the GDR in ${ }^{16} \mathrm{O}$ and about $200 \mathrm{keV}$ for the GQR in the same nucleus. The energy shifts of individual modes are always of the same magnitude. 


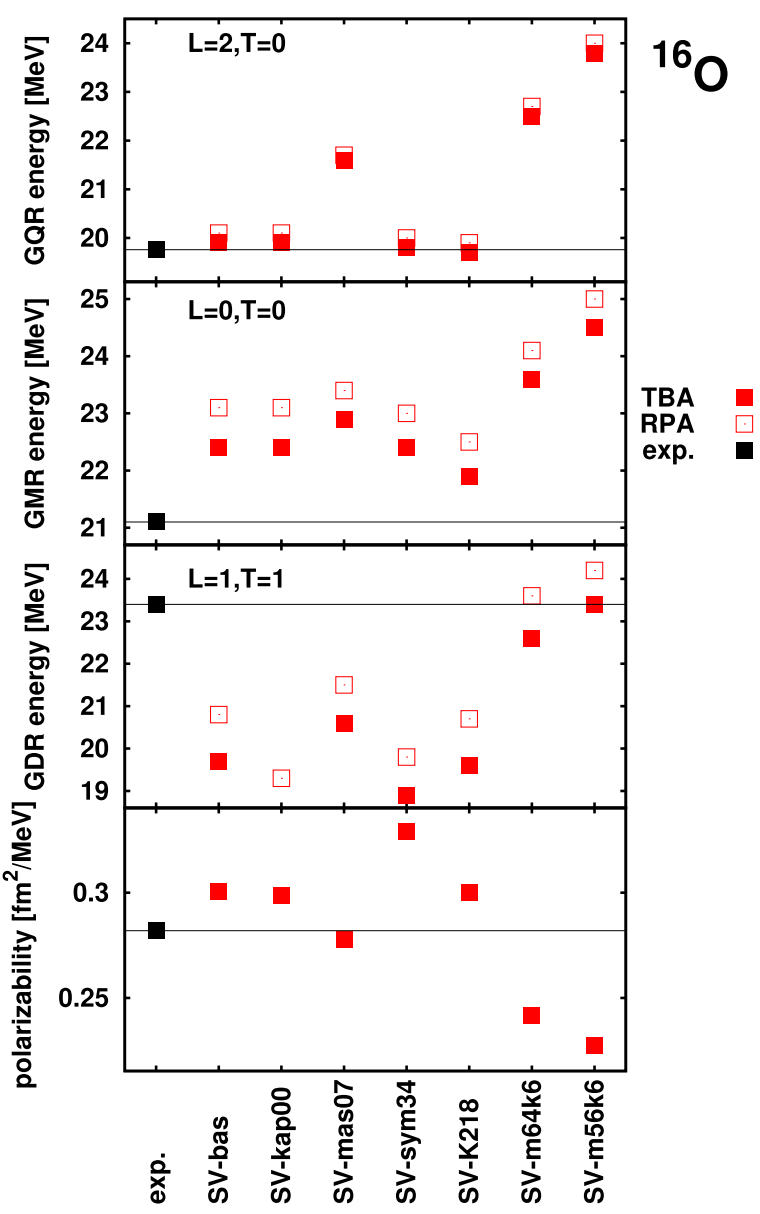

FIG. 11. Same as in Fig. 10, but for ${ }^{16} \mathrm{O}$.

\section{SUMMARY}

The present paper is an extended version of a previous short note [10]. It is concerned with the time-blocking approximation (TBA) which is an extension of the widely used randomphase approximation (RPA) by complex configurations in terms of 1p-1h states coupled to RPA phonons, and it addresses a couple of basic questions in this scheme: proper treatment of the continuum, restoration of stability of ground and excited states, and size of phonon space.

First, we explain here details of the self-consistent continuum TBA, which is a new method for handling the single-particle continuum. This method had been further developed to include also the spin-orbit contribution such that our new calculations are fully self-consistent. We then present numerical results which demonstrate the advantages of the continuum treatment as compared to the conventional treatment in a discrete basis.

The phonon coupling modifies the residual two-body interaction which, in principle, would require one to compute a new, correlated ground state in order to stay consistent and to achieve a stable excitation spectrum with nonimaginary excitation energies. However, this would introduce a double counting because most ground-state correlations are already incorporated in an effective mean-field theory. The problem is solved by the subtraction scheme, subtracting the stationary

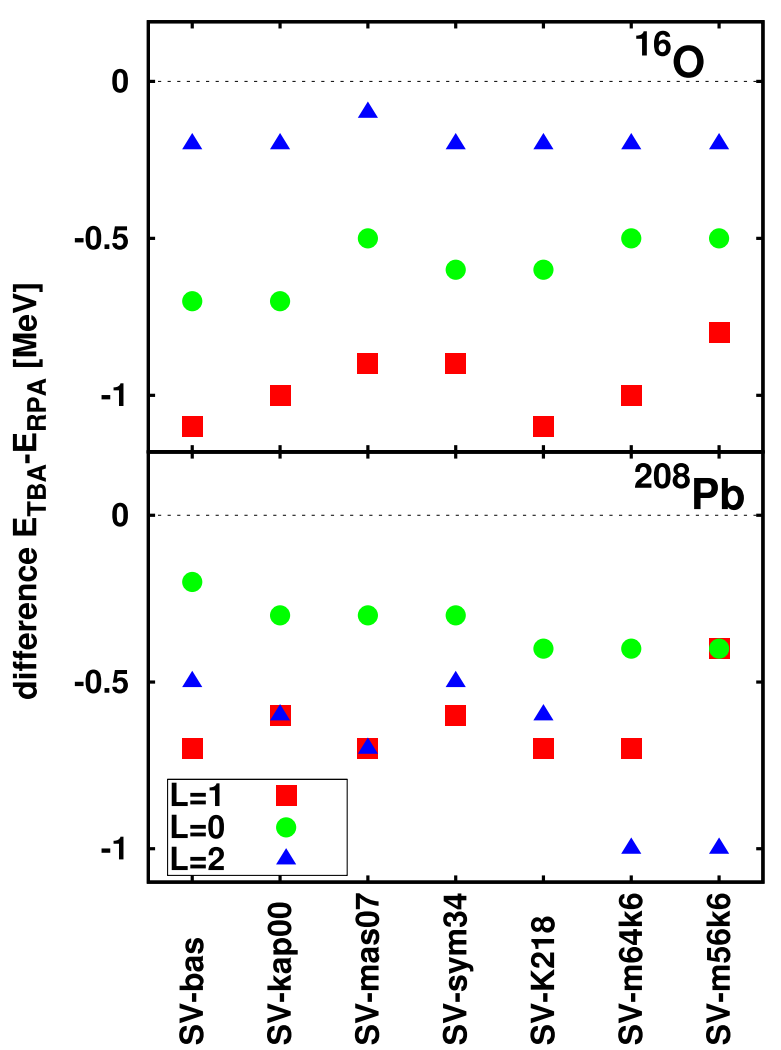

FIG. 12. Difference between TBA and RPA for the giant resonance energies in ${ }^{208} \mathrm{~Pb}$ and ${ }^{16} \mathrm{O}$ for a variety of Skyrme parameter sets as indicated.

(zero-frequency) part of the effective interaction. This leaves the ground state unchanged and delivers stable excitations throughout. It also helps to achieve convergence with phonon number.

A long-standing problem concerns the stability of the TBA with respect to the choice of the number of phonons and the size of the single-particle space. Here we present the results of detailed calculations with systematically scanned numbers of phonons. An important result is that the energies and widths are stable over a large range if the subtraction method is included in the TBA. This identifies a window of phonon numbers where the results are robust.

We obtain that the main qualitative differences between the TBA and RPA are (i) the fragmentation of the resonances in the TBA producing the spreading width and (ii) the downward shift of the peak resonance energies in the TBA.

Having a well tested numerical scheme for (continuum) RPA and TBA at hand, we investigate the dependence of the three main giant resonances on the basic properties of a Skyrme parameter set: incompressibility, isoscalar effective mass, symmetry energy, and TRK sum rule enhancement. And we do that for RPA in comparison to TBA. TBA generally down-shifts the peak resonance energies by up to $1 \mathrm{MeV}$. The shift is about the same for all parameter sets for a given mode and nucleus. It differs for the three modes and also depends on the nucleus. Although the results show a reasonable general agreement with the data, a parameter set which is able to 
describe equally well all three resonance modes in heavy as well as light nuclei has not been found. For nuclei heavier than ${ }^{40} \mathrm{Ca}$, however, there are several effective interactions (SV-bas, SV-mass07, SV-K218) which, when used in TBA, produce spectral strength distributions in fair agreement with the data.

\section{ACKNOWLEDGMENTS}

This work has been supported by contract Re322-13/1 from the DFG. N.L. and V.T. acknowledge St. Petersburg State University for research grant 11.38.648.2013. N.L. acknowledges St. Petersburg State University for research grant 11.38.193.2014. We thank S. P. Kamerdzhiev for fruitful discussions and Dave Youngblood for providing us with experimental data. This research was supported by the Resource Center "Computer Center" of SPbU.

\section{APPENDIX: CONTINUUM IN A DISCRETE BASIS REPRESENTATION}

In the RPA and TBA the response function $R(\omega)$ is a solution of the Bethe-Salpeter equations (9) and (17), respectively. The propagator $R^{(0)}(\omega)$ in these equations in the discrete basis representation has the form

$$
\begin{aligned}
& R_{p h, p^{\prime} h^{\prime}}^{(0)}(\omega)=-\frac{\delta_{p p^{\prime}} \delta_{h^{\prime} h}}{\omega-\varepsilon_{\mathrm{ph}}}, \\
& R_{h p, h^{\prime} p^{\prime}}^{(0)}(\omega)=\frac{\delta_{p^{\prime} p} \delta_{h h^{\prime}}}{\omega+\varepsilon_{\mathrm{ph}}},
\end{aligned}
$$

where $\varepsilon_{\mathrm{ph}}=\varepsilon_{p}-\varepsilon_{h}$.

Let us represent Eqs. (A1) and (A2) in the form

$$
\begin{aligned}
& R_{p h, p^{\prime} h^{\prime}}^{(0)}(\omega)=-\delta_{h^{\prime} h}\left\langle p\left|G^{\mathrm{MF}(+)}\left(\varepsilon_{h}+\omega\right)\right| p^{\prime}\right\rangle, \\
& R_{h p, h^{\prime} p^{\prime}}^{(0)}(\omega)=-\delta_{h h^{\prime}}\left\langle p^{\prime}\left|G^{\mathrm{MF}(+)}\left(\varepsilon_{h}-\omega\right)\right| p\right\rangle,
\end{aligned}
$$

where

$$
G^{\mathrm{MF}(+)}(\varepsilon)=G^{\mathrm{MF}}(\varepsilon)-\sum_{h} \frac{|h\rangle\langle h|}{\varepsilon-\varepsilon_{h}},
$$

$G^{\mathrm{MF}}(\varepsilon)$ is the single-particle mean-field Green function, $|p\rangle$ and $|h\rangle$ are the single-particle wave functions of particles and holes. The superscript $(+)$ in the notation $G^{\mathrm{MF}(+)}(\varepsilon)$ means that this function has the poles only above Fermi level. The equivalence of Eqs. (A1)-(A2) and (A3)-(A4) follows from the spectral expansion

$$
G^{\mathrm{MF}}(\varepsilon)=\sum_{h} \frac{|h\rangle\langle h|}{\varepsilon-\varepsilon_{h}}+\sum_{p} \frac{|p\rangle\langle p|}{\varepsilon-\varepsilon_{p}}
$$

and the orthonormality of the wave functions of the discrete basis.

The discrete basis in this scheme is defined as a complete set of solutions of the Schrödinger equation with the box boundary conditions (b.b.c.). Let us introduce another complete set of solutions of this equation obtained by imposing continuum wave boundary conditions (c.b.c.). This set includes a finite number of the discrete states of holes and particles and a particle continuum. Respective mean-field Green functions and the single-particle states will be denoted as $\tilde{G}^{\mathrm{MF}}(\varepsilon),|\tilde{p}\rangle$, and $|\tilde{h}\rangle$.

The method of inclusion of the continuum in the discrete basis representation consists of the replacement of the uncorrelated particle-hole propagator $R^{(0)}(\omega)$ in Eqs. (9) and (17) by the propagator $\tilde{R}^{(0)}(\omega)$, which is defined by the formulas

$$
\begin{aligned}
\tilde{R}_{p h, p^{\prime} h^{\prime}}^{(0)}(\omega) & =-\delta_{h^{\prime} h}\left\langle p\left|\tilde{G}^{\mathrm{MF}(+)}\left(\varepsilon_{h}+\omega\right)\right| p^{\prime}\right\rangle, \\
\tilde{R}_{h p, h^{\prime} p^{\prime}}^{(0)}(\omega) & =-\delta_{h h^{\prime}}\left\langle p^{\prime}\left|\tilde{G}^{\mathrm{MF}(+)}\left(\varepsilon_{h}-\omega\right)\right| p\right\rangle, \\
\tilde{G}^{\mathrm{MF}(+)}(\varepsilon) & =\tilde{G}^{\mathrm{MF}}(\varepsilon)-\sum_{\tilde{h}} \frac{|\tilde{h}\rangle\langle\tilde{h}|}{\varepsilon-\varepsilon_{\tilde{h}}} .
\end{aligned}
$$

Equations (A7)-(A8) are obtained from Eqs. (A3)-(A4) by the replacement of the function $G^{\mathrm{MF}(+)}(\varepsilon)$ by the function $\tilde{G}^{\mathrm{MF}(+)}(\varepsilon)$. The Green function $\tilde{G}^{\mathrm{MF}}(\varepsilon)$ in Eq. (A9) is calculated in the coordinate representation via the regular and irregular solutions of the Schrödinger equation (with c.b.c.) by means of the known technique [35]. The matrix elements of $\tilde{G}^{\mathrm{MF}(+)}(\varepsilon)$ are calculated with particle wave functions $|p\rangle$ and $\left|p^{\prime}\right\rangle$ of the discrete basis. Thus, the RPA and the TBA equations (9) and (17) are solved in the discrete basis representation. However, in contrast to the initial uncorrelated $p h$ propagator $R^{(0)}(\omega)$, the propagator $\tilde{R}^{(0)}(\omega)$ does not contain the discrete poles $\omega= \pm \varepsilon_{\mathrm{ph}}$ corresponding to the transitions between the hole states and the discrete particle states with positive energies, since these states are replaced by the continuum included in the Green function $\tilde{G}^{\mathrm{MF}}(\varepsilon)$.

This method recovers the exact method [35] of treatment of the continuum in the coordinate representation if the discrete basis is sufficiently complete and the radius of the box is sufficiently large to ensure the equality $|h\rangle=|\tilde{h}\rangle$.

As a criterion of the validity of this equality we choose the absolute value of the difference between the energies of the hole states calculated with continuum wave boundary and box boundary conditions, respectively: $\Delta \varepsilon_{h}=\varepsilon_{h}-\varepsilon_{\tilde{h}}$. In all our calculations (with $R_{\text {box }}=15 \mathrm{fm}$ for ${ }^{16} \mathrm{O},{ }^{40} \mathrm{Ca}$, and ${ }^{48} \mathrm{Ca}$ and $R_{\text {box }}=18 \mathrm{fm}$ for ${ }^{132} \mathrm{Sn}$ and ${ }^{208} \mathrm{~Pb}$ ) we have $\max \left|\Delta \varepsilon_{h}\right| \lesssim$ $10^{-5} \mathrm{MeV}$.

However, the following should be noted. The wave functions of the discrete basis $|p\rangle$ and $|h\rangle$ calculated with the b.b.c form a complete set only inside the box. This fact does not prevent the use of the method described above in the RPA and TBA with the short-range residual interaction (e.g., with the Skyrme forces) due to the natural radial cutoff introduced in the matrix elements by the hole wave functions. This method is also applicable if the direct term of the long-range Coulomb interaction is included because the particle wave functions in the matrix elements of this term are always multiplied by the hole functions taken in the same space point. But the situation is different in the case of the matrix elements of the exact exchange term of the Coulomb interaction where the radial cutoff is absent and therefore the basis should be complete in the whole $r$-space. In our calculations the exchange Coulomb term is treated within the local Slater approximation, so this problem does not arise. 
[1] D. Vretenar, A. V. Afanasjev, G. Lalazissis, and P. Ring, Phys. Rep. 409, 101 (2005).

[2] M. Bender, P.-H. Heenen, and P.-G. Reinhard, Rev. Mod. Phys. 75, 121 (2003).

[3] S. Goriely, M. Samyn, P. H. Heenen, J. M. Pearson, and F. Tondeur, Phys. Rev. C 66, 024326 (2002).

[4] M. Kortelainen, T. Lesinski, J. Moré, W. Nazarewicz, J. Sarich, N. Schunck, M. V. Stoitsov, and S. Wild, Phys. Rev. C 82, 024313 (2010).

[5] J. A. Maruhn, P.-G. Reinhard, P. D. Stevenson, and A. S. Umar, Comput. Phys. Commun. 185, 2195 (2014).

[6] S. Drożdż, S. Nishizaki, J. Speth, and J. Wambach, Phys. Rep. 197, 1 (1990).

[7] J. S. Dehesa, S. Krewald, J. Speth, and A. Faessler, Phys. Rev. C 15, 1858 (1977).

[8] V. I. Tselyaev, Sov. J. Nucl. Phys. 50, 1252 (1989).

[9] V. I. Tselyaev, Phys. Rev. C 75, 024306 (2007).

[10] N. Lyutorovich, V. Tselyaev, J. Speth, S. Krewald, F. Grümmer, and P.-G. Reinhard, Phys. Lett. B 749, 292 (2015).

[11] S. Kamerdzhiev, J. Speth, G. Tertychny, and V. Tselyaev, Nucl. Phys. A 555, 90 (1993).

[12] S. Kamerdzhiev, J. Speth, and G. Tertychny, Phys. Rep. 393, 1 (2004).

[13] E. V. Litvinova and V. I. Tselyaev, Phys. Rev. C 75, 054318 (2007).

[14] B. L. Berman and S. C. Fultz, Rev. Mod. Phys. 47, 713 (1975).

[15] P. Klüpfel, P.-G. Reinhard, T. J. Bürvenich, and J. A. Maruhn, Phys. Rev. C 79, 034310 (2009).

[16] J. Erler, P. Klüpfel, and P.-G. Reinhard, J. Phys. G 38, 033101 (2011).

[17] J. Erler, P. Klüpfel, and P.-G. Reinhard, J. Phys. G 37, 064001 (2010).

[18] S. Abrahamyan et al. (PREX Collaboration), Phys. Rev. Lett. 108, 112502 (2012).

[19] A. Tamii, I. Poltoratska, P. von Neumann-Cosel, Y. Fujita, T. Adachi, C. A. Bertulani, J. Carter, M. Dozono, H. Fujita, K. Fujita, K. Hatanaka, D. Ishikawa, M. Itoh, T. Kawabata, Y. Kalmykov, A. M. Krumbholz, E. Litvinova, H. Matsubara, K. Nakanishi, R. Neveling, H. Okamura, H. J. Ong, B. ÖzelTashenov, V. Y. Ponomarev, A. Richter, B. Rubio, H. Sakaguchi, Y. Sakemi, Y. Sasamoto, Y. Shimbara, Y. Shimizu, F. D. Smit, T. Suzuki, Y. Tameshige, J. Wambach, R. Yamada, M. Yosoi, and J. Zenihiro, Phys. Rev. Lett. 107, 062502 (2011).

[20] D. Savran, M. Elvers, J. Endres, M. Fritzsche, B. Löher, N. Pietralla, V. Y. Ponomarev, C. Romig, L. Schnorrenberger, K. Sonnabend, and A. Zilges, Phys. Rev. C 84, 024326 (2011).

[21] C. J. Horowitz and J. Piekarewicz, Phys. Rev. Lett. 86, 5647 (2001).

[22] N. Lyutorovich, V. I. Tselyaev, J. Speth, S. Krewald, F. Grümmer, and P.-G. Reinhard, Phys. Rev. Lett. 109, 092502 (2012).
[23] N. Lyutorovich, V. Tselyaev, J. Speth, S. Krewald, and P.-G. Reinhard, arXiv:1602.00862 [Phys. At. Nucl. (to be published)].

[24] G. E. Brown, Unified Theory of Nuclear Models and Forces, 3rd ed. (North-Holland, Amsterdam, 1971).

[25] J. Speth, E. Werner, and W. Wild, Phys. Rep. 33, 127 (1977).

[26] J. P. Jeukenne, A. Lejeune, and C. Mahaux, Phys. Rep. 25, 83 (1976).

[27] F. Grümmer and J. Speth, J. Phys. G: Nucl. Part. Phys. 32, R193 (2006)

[28] J. Wambach, V. Mishra, and L. Chu-Hsia, Nucl. Phys. A 380, 285 (1982).

[29] S. P. Kamerdzhiev, G. Y. Tertychny, and V. I. Tselyaev, Fiz. Elem. Chastits At. Yadra 28, 333 (1997) [Phys. Part. Nucl. 28, 134 (1997)]

[30] C. Toepffer and P.-G. Reinhard, Ann. Phys. (N.Y.) 181, 1 (1988).

[31] V. I. Tselyaev, Phys. Rev. C 88, 054301 (2013).

[32] J. R. Stone and P.-G. Reinhard, Prog. Part. Nucl. Phys. 58, 587 (2007)

[33] W. D. Myers, Droplet Model of Atomic Nuclei (IFI/Plenum, New York, 1977).

[34] W. Nazarewicz, P.-G. Reinhard, W. Satula, and D. Vretenar, Eur. Phys. J. A 50, 20 (2014).

[35] S. Shlomo and G. Bertsch, Nucl. Phys. A 243, 507 (1975).

[36] F. Tondeur, M. Brack, M. Farine, and J. M. Pearson, Nucl. Phys. A 420, 297 (1984).

[37] V. Tselyaev, J. Speth, S. Krewald, E. Litvinova, S. Kamerdzhiev, N. Lyutorovich, A. Avdeenkov, and F. Grümmer, Phys. Rev. C 79, 034309 (2009).

[38] O. Bohigas, A. Lane, and J. Martorell, Phys. Rep. 51, 267 (1979).

[39] V. I. Tselyaev, J. Comp. Appl. Math. 170, 103 (2004).

[40] P. Klüpfel, J. Erler, P.-G. Reinhard, and J. A. Maruhn, Eur. Phys. J. A 37, 343 (2008).

[41] T. Nakatsukasa and K. Yabana, Phys. Rev. C 71, 024301 (2005).

[42] V. De Donno, G. Co', M. Anguiano, and A. M. Lallena, Phys. Rev. C 83, 044324 (2011).

[43] Y.-W. Lui, H. L. Clark, and D. H. Youngblood, Phys. Rev. C 64, 064308 (2001).

[44] B. S. Ishkhanov, I. M. Kapitonov, E. I. Lileeva, E. V. Shirokov, V. A. Erokhova, M. A. Yolkin, and A. V. Izotova, preprint INP MSU 2002-27/711.

[45] M. R. Anders, S. Shlomo, T. Sil, D. H. Youngblood, Y.-W. Lui, and Krishichayan, Phys. Rev. C 87, 024303 (2013).

[46] V. A. Erokhova, M. A. Elkin, A. V. Izotova, B. S. Ishkhanov, L. M. Kapitonov, E. I. Lileeva, and E. V. Shirokov, Izv. Ross. Akad. Nauk. Ser. Fiz. 67, 1479 (2003).

[47] K. Knöpfle, G. Wagner, C. Mayer-Böricke, M. Rogge, and P. Turek, Phys. Lett. B 74, 191 (1978).

[48] S. N. Belyaev, O. V. Vasiliev, V. V. Voronov, A. A. Nechkin, V. Y. Ponomarev, and V. A. Semenov, Phys. Atom. Nucl. 58, 1883 (1995).

[49] D. H. Youngblood, Y.-W. Lui, H. L. Clark, B. John, Y. Tokimoto, and X. Chen, Phys. Rev. C 69, 034315 (2004). 